Journal of Computational Neuroscience 18, 205-227, 2005 (C) 2005 Springer Science + Business Media, Inc. Manufactured in The Netherlands.

\title{
A Continuous Attractor Network Model Without Recurrent Excitation: Maintenance and Integration in the Head Direction Cell System
}

\author{
CHRISTIAN BOUCHENY ${ }^{\dagger}$ \\ Laboratory of Physiology of Perception and Action, CNRS-Collège de France, 11 pl. M. Berthelot, \\ 75005 Paris, France \\ NICOLAS BRUNEL \\ Laboratory of Neurophysics and Physiology of the Motor System, CNRS-University Paris 5, 45 rue des Saints \\ Pères, 75270 Paris, Cedex 06, France \\ ANGELO ARLEO $*, \dagger$ \\ Laboratory of Physiology of Perception and Action, CNRS-Collège de France, 11 pl. M. Berthelot, \\ 75005 Paris, France \\ angelo.arleo@csl.sony.fr
}

Received March 9, 2004; Revised July 20, 2004; Accepted November 4, 2004

Action Editor: David Terman

\begin{abstract}
Motivated by experimental observations of the head direction system, we study a three population network model that operates as a continuous attractor network. This network is able to store in a short-term memory an angular variable (the head direction) as a spatial profile of activity across neurons in the absence of selective external inputs, and to accurately update this variable on the basis of angular velocity inputs. The network is composed of one excitatory population and two inhibitory populations, with inter-connections between populations but no connections within the neurons of a same population. In particular, there are no excitatory-to-excitatory connections. Angular velocity signals are represented as inputs in one inhibitory population (clockwise turns) or the other (counterclockwise turns). The system is studied using a combination of analytical and numerical methods. Analysis of a simplified model composed of threshold-linear neurons gives the conditions on the connectivity for (i) the emergence of the spatially selective profile, (ii) reliable integration of angular velocity inputs, and (iii) the range of angular velocities that can be accurately integrated by the model. Numerical simulations allow us to study the proposed scenario in a large network of spiking neurons and compare their dynamics with that of head direction cells recorded in the rat limbic system. In particular, we show that the directional representation encoded by the attractor network can be rapidly updated by external cues, consistent with the very short update latencies observed experimentally by Zugaro et al. (2003) in thalamic head direction cells.
\end{abstract}

Keywords: head direction cells, continuous attractor networks, integrate-and-fire spiking neurons

\footnotetext{
* To whom all correspondence should be addressed.

${ }^{\dagger}$ Current address: Neuroscience Group, Sony Computer Science Laboratory, 6 rue Amyot, 75005 Paris, France.
} 


\section{Introduction}

Continuous attractor network models (Amari, 1977; Ermentrout, 1998) have been proposed to account for selectivity properties of sensory (Ben-Yishai et al., 1995; Somers et al., 1995; Hansel and Sompolinsky, 1998) and motor (Lukashin and Georgopoulos, 1993) systems, for maintenance of a continuous variable in working memory in prefrontal and parietal cortices (Camperi and Wang, 1998; Compte et al., 2000; Laing and Chow, 2001; Gutkin et al., 2001), and for properties of hippocampal place cells (Tsodyks and Sejnowski, 1995; Samsonovich and McNaughton, 1997; Redish and Touretzky, 1997; Battaglia and Treves, 1998; Kali and Dayan, 2000).

Head direction (HD) cells have been hypothesized to constitute yet another system with continuous attractor dynamics (Skaggs et al., 1995; Zhang, 1996; Redish et al., 1996; Goodridge and Touretzky, 2000; Degris et al., 2004). HD cells are observed in a network of structures centered on the rat limbic system. The discharge of these neurons is correlated with the direction of the head of the animal in the azimuthal plane. Each HD cell is selective for one specific 'preferred' direction, regardless of the animal's ongoing behavior and position (Taube, 1998). The response curve of a HD cell (obtained by plotting its mean firing rate as a function of the directional heading) can be approximated by means of a Gaussian tuning profile. The preferred directions of all HD cells are evenly distributed over $360^{\circ}$ such that the HD cell system might work as an allocentric neural compass (Taube, 1995). If the head of the animal remains motionless and oriented in a given direction $\theta$, the sub-population of HD cells with preferred directions close to $\theta$ remains active, demonstrating the persistence of the HD neural activity pattern in stationary conditions.

Although the responses of HD cells are anchored on stable visual cues of the environment (Taube, 1998), the intrinsic dynamics of the HD cell system seems to rely upon the integration of self-motion inertial signals (e.g., vestibular information). During head turns (both in light and in darkness conditions) the ensemble activity profile, determined by the sub-population of HD cells active at a particular time, could vary according to the instantaneous head angular velocity. Thus, besides pure maintenance, the HD cell system seems to have the additional property of integration of head velocity signals and it provides an ongoing memory trace of the direction of the head.
HD cells were first electrophysiologically recorded in the rat postsubiculum (PoSC) (Ranck, 1984; Taube et al., 1990a), and then found in numerous other structures including the laterodorsal thalamic nucleus (Mizumori and Williams, 1993), the dorsal striatum (Wiener, 1993), the retrosplenial cortex (Chen et al., 1994), the anterodorsal thalamic nucleus (ADN) (Taube, 1995; Blair and Sharp, 1995), the lateral mammillary nucleus (LMN) (Stackman and Taube, 1998), and the dorsal tegmental nucleus (DTN) (Sharp et al., 2001).

Although the actual mechanisms underlying the generation of the HD signal are still unclear, anatomical and lesion data suggest that LMN and DTN might constitute an essential sub-circuit of the HD cell system for generating and maintaining the directional signal (Blair et al., 1998; Bassett and Taube, 2001b; Taube and Bassett, 2003). This would yield the following ascending processing scheme: $\mathrm{DTN} \rightarrow \mathrm{LMN} \rightarrow$ ADN $\rightarrow$ PoSC. Lesions to DTN disrupt the directional selectivity in ADN (Bassett and Taube, 2001a). Bilateral lesions of LMN abolish the HD signal in ADN (Blair et al., 1998). The directional selectivity of PoSC cells is seriously impaired when lesioning ADN (Goodridge and Taube, 1997), but lesions of the PoSC leave the signal in ADN largely intact (Goodridge and Taube, 1997). A further evidence for the above processing pathway is provided by a series of studies that have investigated the temporal properties of $\mathrm{HD}$ cells in $\mathrm{LMN}, \mathrm{ADN}$, and PoSC. During head turns, LMN neurons tend to anticipate the future head direction by approximately 40-75 ms (Stackman and Taube, 1998; Blair et al., 1998), ADN cells show a smaller anticipatory time delay of about $25 \mathrm{~ms}$ (Taube and Muller, 1998; Cho and Sharp, 2001), and PoSC cells tend to encode the current directional heading (Blair and Sharp, 1995). The DTNLMN circuit seems also well suited to account for the additional property of the HD cell system of integrating the head angular velocity. The DTN receives ascending inputs from the medial vestibular nucleus, directly and indirectly via the nucleus prepositus hypoglossi (Liu et al., 1984; Bassett and Taube, 2001b), and both DTN and LMN contain neurons whose activity is correlated with the angular velocity of the head in the horizontal plane (Bassett and Taube, 2001b).

Several investigators have proposed one-dimensional continuous attractor networks to model the HD cell system (Skaggs et al., 1995; Zhang, 1996; Redish et al., 1996; Goodridge and Touretzky, 2000; Xie et al., 2002; Degris et al., 2004). As in most 
continuous attractor systems, these models implement a recurrent neural network in which cells representing similar states (i.e., neighboring orientations in the one-dimensional directional state space) are coupled by strong excitatory collaterals, whereas units representing distant states strongly inhibit each other. The intrinsic dynamics of the interaction between excitatory and inhibitory signals generates a center-surround attractor scheme and allows the system to settle down to stable (self-sustained) states where sub-populations of HD neurons with similar preferred directions are active while others remain silent (sometimes called 'bump' states).

In these models, the integration of the head angular velocity is achieved by introducing an asymmetry in the center-surround attractor dynamics, which makes the bump of activity shift over the continuous directional state space. To relate this asymmetric component to head rotations, Zhang (1996) and Redish et al. (1996) modulate the synaptic weights by means of angular velocity signals, whereas Goodridge and Touretzky (2000) utilize synapses with a non-linearity that is tuned to yield a perfect integration of angular velocity signals. Skaggs et al. (1995) hypothesize the existence of two groups of 'rotation cells' that receive vestibular inputs and fire as a function of the magnitude of the angular velocity. One group is responsive for clockwise head turns, the other for counterclockwise turns. The two groups project asymmetrically to the left and to the right of the HD bump of activity, respectively. However, Skaggs et al. (1995) did not simulate any model system to check the feasibility of this scenario. Blair et al. (1998) and Degris et al. (2004) realized a neural implementation of this scenario by proposing an attractor-integrator circuit modeling the LMN-DTN system. Finally, Xie et al. (2002) studied a two population network of left and right units, and showed that with appropriate connections and linear synapses, the network can integrate the inputs with good accuracy in a large velocity range.

All the aforementioned models rely on recurrent excitation to sustain persistent activity in HD neurons. However, anatomical data show no evidence of recurrent excitatory collaterals in the structures of the HD cell system that seem to give rise to the selfsustained signal dynamics, the lateral mammillary nucleus (LMN) and the dorsal tegmental nucleus (DTN) (Allen and Hopkins, 1988, 1989). On the other hand, reciprocal connections between LMN and DTN have been demonstrated experimentally. Anatomical stud- ies suggest that LMN receives ascending inhibitory (GABAergic) afferents from DTN (Shibata, 1987; Allen and Hopkins, 1989; Gonzalo-Ruiz et al., 1992; Wirtshafter and Stratford, 1993) and that, in turn, LMN sends descending excitatory efferents back to DTN (Allen and Hopkins, 1989, 1990). Finally, electrophysiological data suggest that a large fraction of cells in DTN are selective for angular velocity in either clockwise or counterclockwise directions (Bassett and Taube, 2001b; Sharp et al., 2001). These cells could provide the basis for the 'rotation cells' postulated by Skaggs et al. (1995).

Taken together, this anatomical and electrophysiological data motivate investigations of alternative attractor models in which no recurrent excitation is present. Rubin et al. (2001) studied a model of ADN with excitatory thalamocortical (TC) cells and inhibitory thalamic reticular (RE) cells with no recurrent excitatory connections, and showed that a bump state could be sustained thanks to a combination of postinhibitory rebound in TC cells, localized $\mathrm{GABA}_{A}$ and more widespread $\mathrm{GABA}_{B}$ connections from RE to TC cells. Song and Wang (2003) introduced a model with three populations, one excitatory ('LMN') and two inhibitory ('left DTN' and 'right DTN'), and showed, using numerical simulations, that a bump state could be stabilized in such a model with proper wiring between excitatory and inhibitory populations, in absence of recurrent excitation.

The goal of this paper is to analyze in detail the model introduced by Song and Wang (2003). The architecture of the model is shown in Fig. 1. The model includes reciprocal connections between LMN and DTN, whose existence has been demonstrated experimentally. It assumes the existence of two DTN sub-populations, one selective for clockwise turns, the other selective for counterclockwise turns. Finally, the model also assumes that these two DTN networks mutually inhibit each other. As we will see later, such connections are not necessary for the model to operate, but they expand greatly the performance of the system in terms of angular velocity integration. As far as we know, there is no experimental evidence for such a functional lateralization within the rat DTN, nor for the presence of DTN intrastructural inhibition. However, Sharp et al. (2001) report that DTNs in the left-and right-hemispheres exhibit reciprocal inhibitory projections, and show some preliminary electrophysiological data suggesting a tendency for DTN cells to be selective for ipsiversive head rotations. 


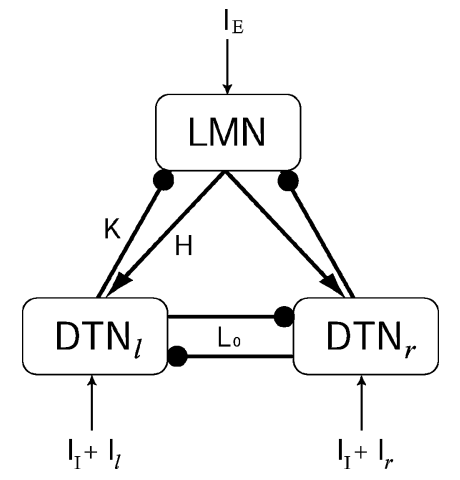

Figure 1. The attractor-integrator model includes a population of excitatory directional units in the lateral mammillary nucleus (LMN) and two networks of inhibitory neurons in the dorsal tegmental nucleus $\left(\mathrm{DTN}_{l, r}\right)$. Arrows and circles indicate excitatory and inhibitory synapses, respectively. The circuit does not contain recurrent excitatory collaterals. LMN receives an external input current $I_{E}$, while $\mathrm{DTN}_{l, r}$ receive modulatory external inputs $I_{I}+I_{l, r}$ providing the system with the head angular velocity signal. (The reader is referred to Appendix A for a table of all abbreviations and variables used in this paper).

The paper is organized as follows. We first study the simplest circuits that exhibit bistability in spite of the absence of recurrent excitation: a model with mutually coupled inhibitory populations, and a model with four populations, two excitatory and two inhibitory. Then, we develop a mathematical analysis of an attractorintegrator model with the architecture of Fig. 1 by using analog (firing rate) simplified neurons, in the spirit of previous studies with simpler architectures (BenYishai et al., 1995; Hansel and Sompolinsky, 1998; Xie et al., 2002). Our results (Sections 2 and 3) provide the analytical insight to the two-fold problem of maintenance and integration of a HD signal in a model without recurrent excitation. In particular, they allow us to identify the conditions on the connectivity parameters for the emergence of a direction selective response and for a reliable integration of head angular velocity signals.

In Section 4 we derive a more realistic implementation of the model by means of large populations of spiking neurons forming an attractor-integrator network according to the architecture shown in Fig. 1. Numerical simulations allow us to compare the responses of the formal HD cells to those of neurons electrophysiologically observed in LMN and DTN. These simulations confirm the persistence and stability properties of the system and the finding that inhibitory projections between the two DTN populations play an important role in determining the stability of the HD representation. Then, we study the integration property of the system and test it on a set of rat angular velocity profiles. Finally, we investigate how rapidly the directional representation encoded by our attractor network can be updated by reorienting external inputs (e.g., visual cues) and find transient latencies consistent with those observed experimentally by Zugaro et al. (2003) in rat HD neurons.

\section{Bistable Networks Without Recurrent Excitation}

Bistability in neuronal systems is usually assumed to arise through positive coupling between excitatory neurons (recurrent excitation). Positive coupling can also arise in absence of recurrent excitation, due to loops involving two (or in general an even number of) inhibitory connections. The simplest system in which bistability can occur is a pair of mutually inhibitory units. Another possibility with mutual connections between excitatory and inhibitory units is a four population network, with two excitatory and two inhibitory populations. A feedback loop is formed through four synapses, two of which are excitatory and two inhibitory. In both cases, excitatory external inputs are required to obtain active states. The study of these simple networks sheds light on the properties of the more complex network models of Sections 3 and 4, which combine features of these two simplified networks. The four population network is a caricature of the LMN-DTN network with only two possible orientations, with 'iso-orientation' projections from LMN to DTN and 'cross-orientation' projections from DTN to $\mathrm{LMN}$; and the two inhibitory population network helps to understand the effect of mutual DTN couplings on the dynamics of the more complete HD cell model.

\subsection{Two Mutually Inhibitory Units with Excitatory External Inputs}

We consider two populations of inhibitory neurons mutually inhibiting each other with strength $L_{0}$. Both populations receive an external excitatory input current $I_{I}$. The circuit is illustrated in Fig. 2A. The firing rates $f_{i_{1}}$ and $f_{i_{2}}$ of the two populations have a continuous 
A

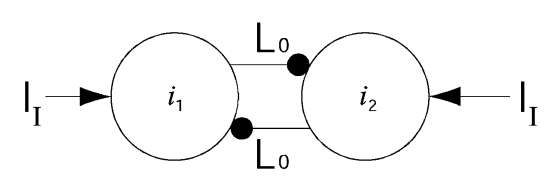

B

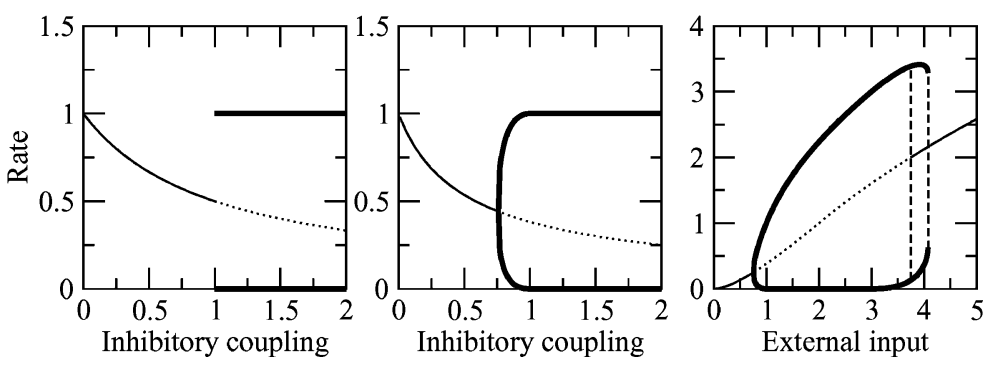

Figure 2. (A) The simplest circuit without recurrent excitation: two mutually inhibitory units each of which receives an external excitatory input $I_{I}$ (black circles and arrows indicate, respectively, inhibitory and excitatory synapses). (B) Bifurcation diagrams (firing rate of populations vs control parameter) of the mutually inhibitory network, for $I_{I}=1$. In all diagrams, thin black line: rates of both populations in a stable symmetric state (both populations at equal rates); thick black lines: rates of both populations in asymmetric states (one population at high rate, the other at low rate); dotted line: unstable symmetric state. The dashed vertical lines indicate the boundaries of the region in which the network has three stable states, one symmetric and two asymmetric states. Left: rates vs inhibitory coupling in the case of a threshold-linear transfer function. Center: rates vs inhibitory coupling in the case of a quadratic/square root transfer function. Right: rates vs $I_{I}$ in the case of a quadratic/square root transfer function and for an inhibitory coupling $L_{0}=1$.

leaky integrator dynamics:

$$
\begin{aligned}
\tau \frac{d f_{i_{1}}(t)}{d t} & =-f_{i_{1}}(t)+\phi(I(t)) \\
& =-f_{i_{1}}(t)+\phi\left(I_{I}-L_{0} f_{i_{2}}(t)\right) \\
\tau \frac{d f_{i_{2}}(t)}{d t} & =-f_{i_{2}}(t)+\phi(I(t)) \\
& =-f_{i_{2}}(t)+\phi\left(I_{I}-L_{0} f_{i_{1}}(t)\right)
\end{aligned}
$$

where $\phi$ is the steady-state 'current-to-rate' neuronal transfer function.

Threshold linear neurons have a firing rate $f$ that depends on the input current $I$ through the relationship $f=\phi(I)=[I]_{+}$, where $[I]_{+}=0$ for $I<0$, and $[I]_{+}=I$ for $I>0$. In this case, such a network has two types of solutions, depending on the strength of the inhibitory coupling $L_{0}$. If $L_{0}<1$, the uniform solution is the only stable solution. The rate of both populations in the uniform solution is

$$
f_{i_{1}}=f_{i_{2}}=\left[\frac{I_{I}}{1+L_{0}}\right]_{+}
$$

When $L_{0}>1$ and $I_{I}>0$, the uniform solution becomes unstable. There is a spontaneous symmetry breaking and one of two possible solutions is selected by the system, either $f_{i_{1}}=I_{I}$ and $f_{i_{2}}=0$, or vice versa. This spontaneous symmetry breaking is shown in Fig. 2B (left panel).

For non-linear response functions, symmetry breaking occurs for $L_{0} \phi^{\prime}>1$ where $\phi^{\prime}$ represents the gain of the neuronal gain function at the uniform firing rate (derivative of the $f$ - $I$ curve $\phi(I)$ with respect to the input current $I$, taken at the firing rate of the uniform solution). For example, if we consider a quadratic/square root transfer function (Brunel, 2003)

$$
\phi(I)= \begin{cases}0 & I<0 \\ I^{2} & I \in[0,1] \\ 2 \sqrt{I-3 / 4} & I>1\end{cases}
$$

we obtain the bifurcation diagram shown in Fig. 2B (center and right panels). Note that in the case of this transfer function, the tuned state emerges continuously from the uniform state, unlike in the case of a thresholdlinear transfer function (compare left and center panels in Fig. 2B). The quadratic/square root transfer function is used here due to its simplicity, and because it shares the qualitative features of the transfer function of many spiking neuron models: (i) the 'quadratic' 
region mimics the typical shape of the $f-I$ curve of a spiking neuron for sub-threshold inputs in presence of noise; (ii) a 'square root' behavior is typically obtained for supra-threshold inputs for a wide class of neurons, the so-called type $I$ neurons (see discussion in Brunel, 2003). Other continuous non-linear transfer functions show the same qualitative behavior (not shown).

\subsection{Two Excitatory and Two Inhibitory Units}

Let $e_{1}$ and $e_{2}$ denote two populations of excitatory neurons and let $i_{1}$ and $i_{2}$ be two populations of inhibitory neurons. The excitatory population $e_{1}$ activates the inhibitory population $i_{1}$, while $e_{2}$ projects onto $i_{2}$. The efficacy of these excitatory projections is $H_{1}$. The populations $i_{1}$ and $i_{2}$ inhibit, respectively, the populations $e_{2}$ and $e_{1}$ with strength $K_{1}$. Both excitatory populations $e_{1}$ and $e_{2}$ receive an external input current $I_{E}$. The circuit is illustrated in Fig. 3A. It can be considered as a simplified version (which can only encode two possible head directions) of the more complete model of Sections 3 and 4 (in which a continuum of head directions can be represented).
The firing rates of the four neural populations evolve according to

$$
\begin{aligned}
\tau \frac{d f_{e_{1}}(t)}{d t} & =-f_{e_{1}}(t)+\phi\left(I_{E}-K_{1} f_{i_{2}}(t)\right) \\
\tau \frac{d f_{e_{2}}(t)}{d t} & =-f_{e_{2}}(t)+\phi\left(I_{E}-K_{1} f_{i_{1}}(t)\right) \\
\tau \frac{d f_{i_{1}}(t)}{d t} & =-f_{i_{1}}(t)+\phi\left(H_{1} f_{e_{1}}(t)\right) \\
\tau \frac{d f_{i_{2}}(t)}{d t} & =-f_{i_{2}}(t)+\phi\left(H_{1} f_{e_{2}}(t)\right)
\end{aligned}
$$

For threshold-linear neurons, such a network has again two types of solutions, depending on the strength of the excitatory-inhibitory loop defined by $K_{1} H_{1}$. If $K_{1} H_{1}<1$, the uniform solution is the only stable solution. For $I_{E}>0$, the firing rate functions are equal to

$$
\begin{gathered}
f_{e_{1}}=f_{e_{2}}=\frac{I_{E}}{1+K_{1} H_{1}} \\
f_{i_{1}}=f_{i_{2}}=\frac{H_{1} I_{E}}{1+K_{1} H_{1}}
\end{gathered}
$$

If $K_{1} H_{1}>1$, the uniform solution becomes unstable. The system settles down to one of two possible solutions, either $f_{e_{1}}=I_{E}, f_{i_{1}}=H_{1} I_{E}$, and $f_{e_{2}}=$ $f_{i_{2}}=0$, or vice versa. A bifurcation diagram showing the firing rates as a function of either $K_{1}$ or $H_{1}$ would look qualitatively as those of Fig. 2.

A

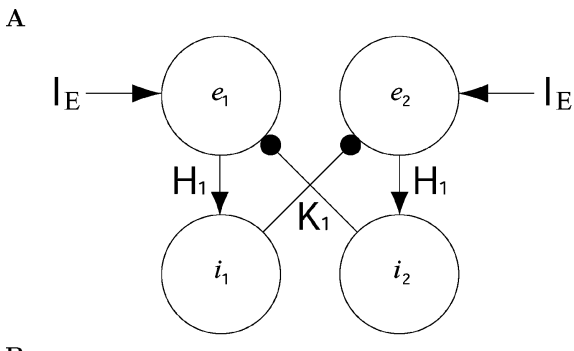

B

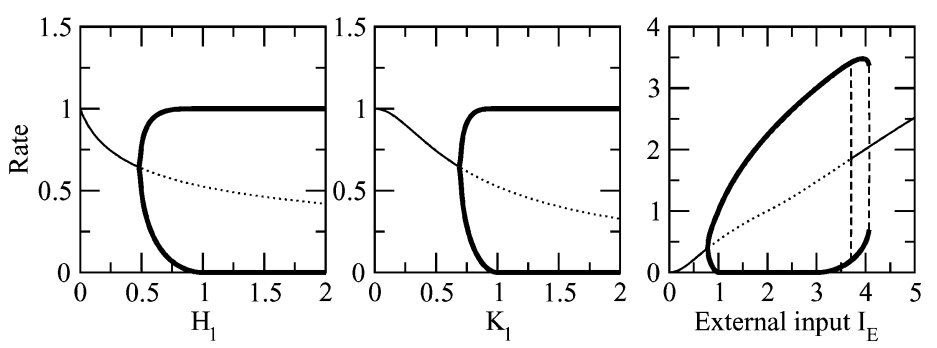

Figure 3. (A) Circuit composed of two excitatory $\left(e_{1,2}\right)$ and two inhibitory $\left(i_{1,2}\right)$ units. $e_{1,2}$ receive an external excitatory current $I_{E}$. (B) Bifurcation diagrams of the excitatory-inhibitory network. Firing rates of the excitatory populations $e_{1}$ and $e_{2}$ are shown as a function of $H_{1}$ (left), $K_{1}$ (center) and $I_{E}$ (right). Parameters: $H_{1}=1$ (except in left), $K_{1}=1$ (except in center), $I_{E}=1$ (except in right). The uniform states (thin solid line) become unstable (dotted line) above a critical value of $H_{1}$ (left) and $K_{1}$ (center), and at intermediate values of $I_{E}$ (right). The asymmetric states (one pair of populations suppressing the other pair) are indicated by thick black lines. At some intermediate level of $I_{E}$, the system is tristable (between the dashed lines in right panel). Note the similarity with the diagrams of the mutually inhibitory network, Fig. 2. 
In the case of non-linear response functions, symmetry breaking occurs for $H_{1} K_{1} \phi_{e}^{\prime} \phi_{i}^{\prime}>1$, where $\phi_{e, i}^{\prime}$ represents the gains of the $e$ and $i$ neuronal gain functions at their uniform firing rate (derivative of the $f-I$ curve $\phi(I)$ with respect to the input current $I$, taken at the firing rate of the uniform solution). The bifurcation diagrams (rates vs $K_{1}, H_{1}$ or $I_{E}$ ) are shown in Fig. 3B.

\section{Ring Attractor Networks Without Recurrent Excitation}

The model presented here is a generalization of previous ring attractor networks (Ben-Yishai et al., 1995, 1997; Zhang, 1996; Redish et al., 1996; Hansel and Sompolinsky, 1998; Goodridge and Touretzky, 2000; Xie et al., 2002; Degris et al., 2004). All these previous studies consider one or two population networks (either one population with couplings that can be either excitatory or inhibitory, or an excitatory-inhibitory network). Here, we investigate the three-population architecture proposed by Song and Wang (2003): one excitatory population (representing the LMN), and two inhibitory populations (representing two distinct populations in the DTN, one selective for clockwise turns, the other selective for counterclockwise turns). In this section, we consider a network of simplified threshold-linear firing rate units. This allows us to obtain analytical expressions for the firing rate profiles of the network in stationary states as well as in traveling states, and to explore systematically the parameter space. Numerical simulations of a network of spiking neurons with a similar architecture are presented in Section 4.

The network architecture is shown in Fig. 1. The network is composed of a population of excitatory directional units with evenly distributed preferred directions ('LMN' or ' $e$ ' network), and two populations of inhibitory directional neurons with evenly distributed preferred directions ('left DTN' or ' $l$ ', and 'right DTN' or ' $r$ '). All excitatory neurons receive an external input current $I_{E}$ as well as inhibitory afferents from both inhibitory networks. The projection from a neuron in left DTN with preferred direction $\theta^{\prime}$ to a neuron in LMN with label $\theta$ has a synaptic efficacy defined by $K\left(\theta-\theta^{\prime}-\pi+\alpha\right)$, where $\alpha$ is an angular offset, and $K(\theta)=K_{0}+K_{1} \cos (\theta)$, where $K_{1} \leq K_{0}$. The $\pi$ term indicates that the $l \rightarrow e$ projections generate crossorientation inhibition. Thus, a left DTN neuron with label $\theta^{\prime}$ inhibits maximally a LMN neuron with label $\theta$ only when $\theta-\theta^{\prime}=\pi-\alpha$. The connection from a right DTN unit with label $\theta^{\prime}$ to a LMN neuron with label $\theta$ is defined by $K\left(\theta-\theta^{\prime}-\pi-\alpha\right)$. That is, the unit inhibits mostly the neuron having a preferred orientation $\pi+\alpha$ away from its preferred direction.

All inhibitory neurons receive an external input current $I_{I}+I_{l}$ and $I_{I}+I_{r}$, respectively, where $I_{I}$ represents a background current to both populations, while $I_{l}$ and $I_{r}$ represent angular velocity-dependent input currents. In addition, both networks receive excitatory afferents from the LMN population according to an iso-orientation connection scheme. The projection from a LMN neuron with preferred direction $\theta$ to a DTN neuron with label $\theta^{\prime}$ is $H\left(\theta-\theta^{\prime}\right)$ with $H(\theta)=H_{0}+H_{1} \cos (\theta)$ and $H_{1} \leq H_{0}$. Finally, left and right DTNs have mutual inhibitory connections with fixed strength $L_{0}$. All neurons are analog units described by an instantaneous firing rate $f_{e, l, r}(\theta, t)$ and a synaptic activation variable $s_{e, l, r}(\theta, t)$, where $\theta$ is the preferred orientation of the neuron and $t$ is time, and $e, l, r$ is the population index ( $e$ : LMN, $l$ : left DTN, $r$ : right DTN). The dynamics of the synaptic activation variables and firing rates of the system is described by the following set of equations:

$$
\begin{aligned}
\tau \dot{s}_{e}(\theta, t) & =-s_{e}(\theta, t)+f_{e}(\theta, t) \\
\tau \dot{s}_{l}(\theta, t) & =-s_{l}(\theta, t)+f_{l}(\theta, t) \\
\tau \dot{s}_{r}(\theta, t) & =-s_{r}(\theta, t)+f_{r}(\theta, t) \\
f_{e}(\theta, t) & =\left[I_{E}(t)-\int_{-\pi}^{\pi} \frac{K\left(\theta-\theta^{\prime}-\pi+\alpha\right) s_{l}\left(\theta^{\prime}, t\right)+K\left(\theta-\theta^{\prime}-\pi-\alpha\right) s_{r}\left(\theta^{\prime}, t\right)}{4 \pi} d \theta^{\prime}\right]_{+} \\
f_{l}(\theta, t) & =\left[I_{I}+I_{l}(t)+\int_{-\pi}^{\pi} \frac{H\left(\theta-\theta^{\prime}\right) s_{e}\left(\theta^{\prime}, t\right)+L_{0} s_{r}\left(\theta^{\prime}, t\right)}{2 \pi} d \theta^{\prime}\right]_{+} \\
f_{r}(\theta, t) & =\left[I_{I}+I_{r}(t)+\int_{-\pi}^{\pi} \frac{H\left(\theta-\theta^{\prime}\right) s_{e}\left(\theta^{\prime}, t\right)-L_{0} s_{l}\left(\theta^{\prime}, t\right)}{2 \pi} d \theta^{\prime}\right]_{+}
\end{aligned}
$$


Hence, the synaptic activation variables are obtained from the firing rates by a simple temporal low-pass filtering, with synaptic time constant $\tau$ (Eqs. (11)-(13)), which is the only time constant in this model (for simplicity, we take excitatory and inhibitory time constants to be identical here). The firing rates are in turn given by a threshold-linear function of the synaptic inputs, which are given by the various synaptic variables convolved by the relevant connection profiles (Eqs. (14)(16)).

Following previous studies (Ben-Yishai et al., 1995, 1997; Hansel and Sompolinsky, 1998; Xie et al., 2002), we define the variables $s_{A 0}(t)$ and $s_{A 1}(t)$ as

$$
\begin{aligned}
s_{A 0}(t) & =\int_{-\pi}^{\pi} \frac{s_{A}(\theta, t) d \theta}{2 \pi} \\
s_{A 1}(t) & =\int_{-\pi}^{\pi} \frac{s_{A}(\theta, t) \cos \left(\theta-\psi_{A}(t)\right) d \theta}{2 \pi} \\
0 & =\int_{-\pi}^{\pi} \frac{s_{A}(\theta, t) \sin \left(\theta-\psi_{A}(t)\right) d \theta}{2 \pi}
\end{aligned}
$$

where the index $A=e, l, r$ defines the neural population corresponding to the variable; $s_{A 0}(t)$ is the average synaptic activation variable of population $A ; s_{A 1}(t)$ is the first Fourier component of the activation variable; and $\psi_{A}(t)$ is the center of mass of the activation variable (location of the peak in the case of a bump solution). Using these variables, the integrals in Eqs. (14)-(16) can be performed explicitly, and the firing rates for the populations $e, l$, and $r$ become:

$$
\begin{aligned}
f_{e}(\theta, t)= & {\left[I_{E}(t)-\frac{K_{0}}{2}\left[s_{l 0}(t)+s_{r 0}(t)\right]\right.} \\
& +\frac{K_{1}}{2}\left[\cos \left(\theta+\alpha-\psi_{l}(t)\right) s_{l 1}(t)+\cos (\theta\right. \\
& \left.\left.\left.-\alpha-\psi_{r}(t)\right) s_{r 1}(t)\right]\right]_{+} \\
f_{l}(\theta, t)= & {\left[I_{I}+I_{l}(t)+H_{0} s_{e 0}(t)-L_{0} s_{r 0}(t)\right.} \\
& \left.+H_{1} \cos \left(\theta-\psi_{e}(t)\right) s_{e 1}(t)\right]_{+} \\
f_{r}(\theta, t)= & {\left[I_{I}+I_{r}(t)+H_{0} s_{e 0}(t)-L_{0} s_{l 0}(t)\right.} \\
& \left.+H_{1} \cos \left(\theta-\psi_{e}(t)\right) s_{e 1}(t)\right]_{+}
\end{aligned}
$$

From Eqs. (11)-(13), the dynamics of the variables $s_{A 0}, s_{A 1}$ and $\psi_{A}$ are given by

$$
\tau \dot{s}_{A 0}(t)=-s_{A 0}(t)+\int_{-\pi}^{\pi} f_{A}(\theta, t) \frac{d \theta}{2 \pi}
$$

$$
\begin{aligned}
\tau \dot{s}_{A 1}(t) & =-s_{A 1}(t)+\int_{-\pi}^{\pi} f_{A}(\theta, t) \frac{\cos \theta d \theta}{2 \pi} \\
\tau \dot{\psi}_{A}(t) s_{A 1}(t) & =\int_{-\pi}^{\pi} f_{A}(\theta, t) \frac{\sin \theta d \theta}{2 \pi}
\end{aligned}
$$

We rewrite the equations for the firing rate profiles as

$$
\begin{array}{r}
f_{A}(\theta, t)=\left[I_{A 0}(t)+I_{A 1}(t) \cos \left(\theta-\phi_{A}(t)\right)\right]_{+} \\
A=e, l, r
\end{array}
$$

where $I_{A 0}(t)$ is the synaptic input averaged over all neurons in population $A$ at time $t, I_{A 1}(t)$ is the first Fourier component of the synaptic input, and $\phi_{A}(t)$ is the instantaneous location of the bump, i.e. neurons with preferred direction $\phi_{A}(t)$ have the peak firing rates of population $A$ at time $t$. The parameters $I_{A 0}(t), I_{A 1}(t)$ and $\phi_{A}(t)$ are given by the system of equations

$$
\begin{aligned}
I_{e 0}(t)= & I_{E}(t)-\frac{K_{0}}{2}\left(s_{l 0}(t)+s_{r 0}(t)\right) \\
I_{l 0}(t)= & I_{I}+I_{l}(t)+H_{0} s_{e 0}(t)-L_{0} s_{r 0}(t) \\
I_{r 0}(t)= & I_{I}+I_{r}(t)+H_{0} s_{e 0}(t)-L_{0} s_{l 0}(t) \\
I_{e 1}(t)= & \frac{K_{1}}{2}\left(\cos \left(\psi_{l}(t)-\alpha-\phi_{e}(t)\right) s_{l 1}\right. \\
& \left.+\cos \left(\psi_{r}(t)+\alpha-\phi_{e}(t)\right) s_{r 1}(t)\right) \\
0= & \sin \left(\psi_{l}(t)-\alpha-\phi_{e}(t)\right) s_{l 1}(t) \\
& +\sin \left(\psi_{r}(t)+\alpha-\phi_{e}(t)\right) s_{r 1}(t) \\
I_{l 1}(t)= & H_{1} s_{e 1}(t) \\
I_{r 1}(t)= & H_{1} s_{e 1}(t) \\
\phi_{l}(t)= & \psi_{e}(t) \\
\phi_{r}(t)= & \psi_{e}(t)
\end{aligned}
$$

To calculate the integrals in Eqs. (23)-(25), we define $\theta_{A}(t)=\arccos \left(-I_{A 0}(t) / I_{A 1}(t)\right)$ as the width of the bump of the firing rate profile $\left(f_{A}(\theta, t)=0\right.$ for $\theta$ outside of the range $\left[\phi_{A}(t)-\theta_{A}(t), \phi_{A}(t)+\theta_{A}(t)\right]$, while $f_{A}(\theta, t)$ is positive within that range). The integrals yield

$$
\begin{aligned}
\int_{-\theta_{A}}^{\theta_{A}} \frac{d \theta}{2 \pi}\left(I_{A 0}+I_{A 1} \cos (\theta)\right) & =I_{A 1} f_{0}\left(\theta_{A}\right) \\
\int_{-\theta_{A}}^{\theta_{A}} \frac{d \theta}{2 \pi}\left(I_{A 0}+I_{A 1} \cos (\theta)\right) \cos \theta & =I_{A 1} f_{1}\left(\theta_{A}\right)
\end{aligned}
$$

where $f_{0}$ and $f_{1}$ are given by

$$
\begin{aligned}
& f_{0}(x)=\frac{1}{\pi}(\sin x-x \cos x) \\
& f_{1}(x)=\frac{1}{2 \pi}\left(x-\frac{\sin 2 x}{2}\right)
\end{aligned}
$$


Finally, the equations governing the dynamical variables $s_{A 0}(t), s_{A 1}(t)$ and $\psi_{A}(t)$ are

$$
\begin{aligned}
\tau \dot{s}_{A 0}(t)= & -s_{A 0}(t)+I_{A 1}(t) f_{0}\left(\theta_{A}(t)\right) \\
\tau \dot{s}_{A 1}(t)= & -s_{A 1}(t) \\
& +I_{A 1}(t) f_{1}\left(\theta_{A}(t)\right) \cos \left(\phi_{A}(t)-\psi_{A}(t)\right)
\end{aligned}
$$

$\tau \dot{\psi}_{A}(t) s_{A 1}(t)=I_{A 1}(t) f_{1}\left(\theta_{A}(t)\right) \sin \left(\phi_{A}(t)-\psi_{A}(t)\right)$

where $I_{A 1}(t), \theta_{A}(t)$ and $\phi_{A}(t)$ are given by Eqs. (27)(35). Equations (40)-(42) completely describe the dynamics of the system for any time-dependent input.

\subsection{Stationary Solutions}

This section considers a stationary situation where $I_{l}=$ $I_{r}=0$, that is the two inhibitory networks receive the same external signal $I_{I}$. We seek a solution to Eqs. (40)(42) with $\psi_{e}=\psi_{l}=\psi_{r}=0, s_{l 0}=s_{r 0} \equiv s_{i 0}, s_{l 1}=$ $s_{r 1} \equiv s_{i 1}, I_{l 0}=I_{r 0} \equiv I_{i 0}, I_{l 1}=I_{r 1} \equiv I_{i 1}, \theta_{l}=$ $\theta_{r} \equiv \theta_{i}$. The synaptic activation variables and the firing rate variables are then

$$
\begin{aligned}
s_{A 0} & =I_{A 1} f_{0}\left(\theta_{A}\right), \quad A=e, i \\
s_{A 1} & =I_{A 1} f_{1}\left(\theta_{A}\right), \quad A=e, i \\
\theta_{A} & =\arccos \left(-\frac{I_{A 0}}{I_{A 1}}\right), \quad A=e, i \\
I_{e 0} & =I_{E}-K_{0} s_{i 0} \\
I_{i 0} & =I_{I}+H_{0} s_{e 0}-L_{0} s_{i 0} \\
I_{e 1} & =K_{1} \cos \alpha s_{i 1} \\
I_{i 1} & =H_{1} s_{e 1}
\end{aligned}
$$

These equations define the firing rate profile of stationary states as a function of seven parameters: $I_{E}, I_{I}, K_{0}, K_{1} \cos \alpha, L_{0}, H_{0}$ and $H_{1}$.

3.1.1. Uniform State. The spatially uniform state has $s_{A 1}=0$ for $A=e, l, r$. The firing rates of the three populations are given by

$$
\begin{aligned}
& s_{e 0}=\left[I_{E}-K_{0} s_{i 0}\right]_{+} \\
& s_{l 0}=s_{r 0} \equiv s_{i 0}=\frac{\left[I_{I}+H_{0} s_{e 0}\right]_{+}}{1+L_{0}}
\end{aligned}
$$

In particular, both excitatory and inhibitory firing rates are non-zero for $I_{E}>K_{0} I_{I} /\left(1+L_{0}\right)$.

3.1.2. Stability of Uniform State. To study the stability of the uniform state, one must compute the time evolution of small perturbations around it. Small perturbations around the uniform state are denoted by $\delta s_{A 0}, \delta s_{A 1}$. We rewrite $\Sigma s_{i 0}=\left(\delta s_{l 0}+\delta s_{r 0}\right) / 2$, $\Delta s_{i 0}=\left(\delta s_{l 0}-\delta s_{r 0}\right) / 2, \Sigma s_{i 1}=\left(\delta s_{l 1}+\delta s_{r 1}\right) / 2$, $\Delta s_{i 1}=\left(\delta s_{l 1}-\delta s_{r 1}\right) / 2$. From Eqs. (40)-(42), the evolution equations for these small perturbations can be obtained:

$$
\begin{aligned}
\tau \frac{d \delta s_{e 0}}{d t} & =-\delta s_{e 0}-K_{0} \Sigma s_{i 0} \\
\tau \frac{d \sum s_{i 0}}{d t} & =-\left(1+L_{0}\right) \sigma s_{i 0}+H_{0} \delta s_{e 0} \\
\tau \frac{d \Delta s_{i 0}}{d t} & =-\left(1-L_{0}\right) \Delta s_{i 0} \\
\tau \frac{d \delta s_{e 1}}{d t} & =-\delta s_{e 1}+\frac{K_{1} \cos (\alpha)}{2} \Sigma s_{i 1} \\
\tau \frac{d \Sigma s_{i 1}}{d t} & =-\Sigma s_{i 1}+\frac{H_{1}}{2} \delta s_{e 1} \\
\frac{d \Delta s_{i 1}}{d t} & =-\Delta s_{i 1}
\end{aligned}
$$

The 6 eigenvalues of the corresponding linear stability matrix can be computed easily:

- The two eigenvalues associated with Eqs. (52) and (53) always have negative real parts.

- From Eq. (54), we obtain that for $L_{0}>1$, initial small differences in activity in left and right populations grow exponentially in time; this is the instability studied in Section 2.1. Hence, the left-right inhibitory network becomes bistable.

- From Eqs. (55) and (56), we obtain that for $H_{1} K_{1} \cos \alpha>4$, the uniform state becomes unstable, and the system converges to a tuned state.

Hence, two types of instabilities of the uniform state may occur. For $L_{0}>1$, the network becomes bistable (due to mutual inhibition between left and right networks). This instability of the uniform state is similar to the instability of the two population network of Section 2.1. For $H_{1} K_{1} \cos \alpha>4$, the network becomes tuned, and has a continuum of attractor states, parameterized by the angular location of the bump. This instability of the uniform state is the equivalent of the instability of the four population network of Section 2.2. 
3.1.3. The Stationary Tuned State. The firing rate profiles in the tuned state beyond the bifurcation at $H_{1} K_{1} \cos \alpha>4$ can be obtained by solving numerically Eqs. (43)-(49). For example, Fig. 4 shows the mean and peak firing rates in all populations as one of the parameters is varied. In the particular example shown in Fig. 4, $L_{0}=0.5, H_{0}=H_{1}=1.5, K_{0}=$ $K_{1}=K, \alpha=\pi / 3, I_{I}=0$, and $I_{E}=10(1+K)$.
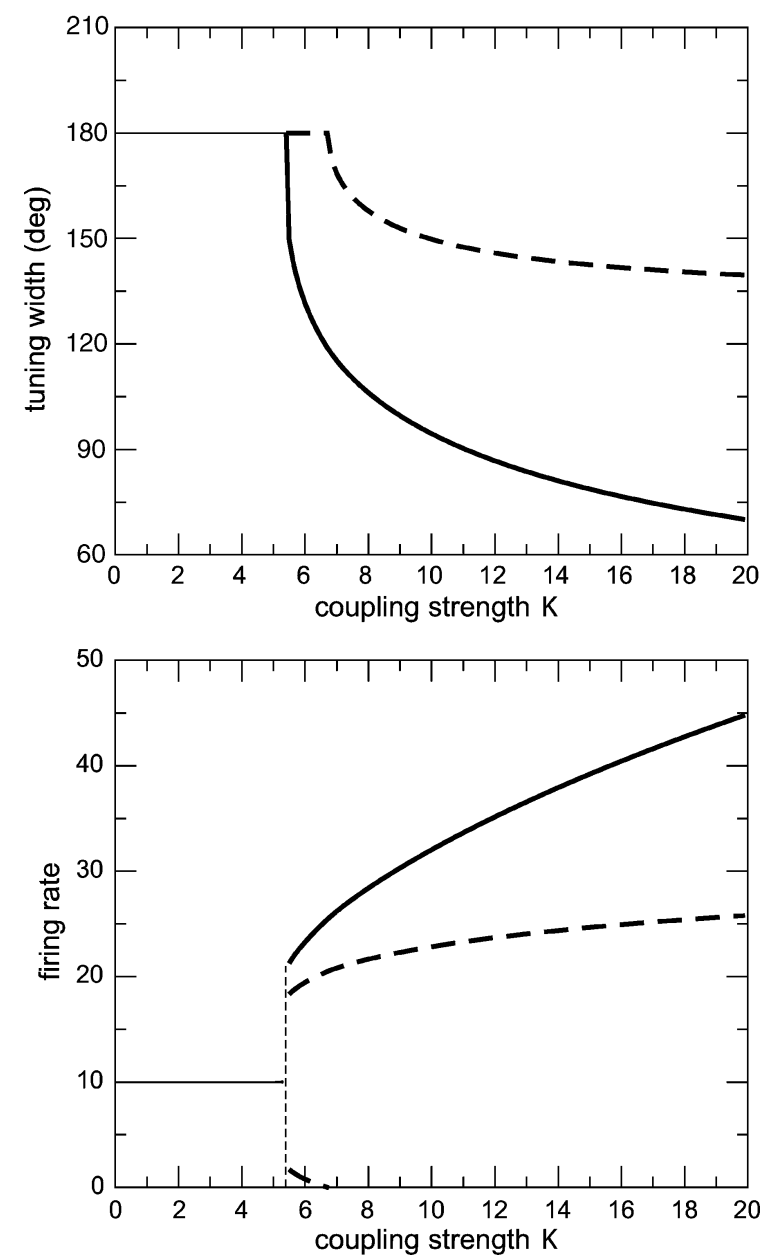

Figure 4. Characteristics of the tuned state vs connection strength $K$. Top: width of the tuned state as a function of $K$. Bottom: peak and background firing rates as a function of $K$ (solid line: excitatory network; dashed line: inhibitory networks; thin lines: uniform state; thick lines: tuned state). For $K<16 / 3$ (marked by the thin dashed line), the uniform state is the only stable stationary state. Above $K=16 / 3$, the tuned state becomes the only stable stationary state. Note that the tuning widths decrease while $K$ is increasing, while the peak firing rates increase with increasing $K$. Other parameters: $L_{0}=0.5, H_{0}=H_{1}=1.5, K_{0}=K_{1}=K, \alpha=\pi / 3, I_{I}=0$, and $I_{E}=10(1+K)$.
The relationship between $I_{E}$ and $K$ ensures that the mean firing rates in the uniform state remain constant as $K$ is varied. Note that the bottom panel of Fig. 4 is the analog of Fig. 3B. The basic mechanism for generating selective attractor states is essentially similar in the two networks: a combination of connections from excitatory to inhibitory neurons with the same preferred direction, and connections from inhibitory to excitatory neurons with opposite preferred directions. Note also that the opposite connectivity scheme ('cross-orientation' from excitatory to inhibitory neurons, 'iso-orientation' from inhibitory to excitatory neurons) would yield qualitatively the same results. The difference between the network of Section 2.2 and the network studied here is that beyond the bifurcation, the first has only two attractors, while the second has a continuum of attractors, parameterized by the angle $\theta$.

Conversely, given the firing rate profiles, one can deduce the connectivity parameters $H_{1}$ and $K_{1} \cos \alpha$ through Eqs. (48) and (49), and two relationships between the remaining five parameters. For example, if we consider a firing profile of width $\theta_{e}=80^{\circ}$ and a peak firing rate $I_{e 0}+I_{e 1}=50 \mathrm{~Hz}$ for the excitatory network $e$, and we take $\theta_{i}=110^{\circ}$ and a peak firing rate $I_{i 0}+I_{i 1}=30 \mathrm{~Hz}$ for the inhibitory networks $l$ and $r$, we obtain $H_{1}=1.89, K_{1} \cos \alpha=7.61$. A set of parameters for which the given firing rate profiles are stable is then $H_{0}=1.89, I_{E}=111, K_{1}=10.7, L_{0}=$ $1.71, I_{I}=0$.

3.1.4. Stability of the Stationary Tuned State. Linear stability analysis of the stationary tuned state can be performed along the same lines as the analysis of the uniform state. The analysis is more complicated because the eigenvalues are no longer obtained by solutions of linear or quadratic equations. They can be obtained by finding numerically the eigenvalues of the stability matrix associated with Eqs. (40)-(42). In particular, we find that, again, a large value of $L_{0}$ leads to a destabilization of the stationary state: one of the two inhibitory populations (left or right) becomes active at high rates and suppresses the activity of the other. As a consequence of the offset in the connections, the bumps in the three populations start to move, even though inputs to left and right populations are equal. Hence, a traveling bump is obtained. The condition for the stationary bump to start moving is $L_{0}>\pi / \theta_{i}$ (see below), to be compared with the condition $L_{0}>1$ for the stationary state. 


\subsection{Traveling Bump Solutions}

When the external inputs to the inhibitory networks $l$ and $r$ differ, i.e. $I_{l} \neq I_{r}$, the stationary attractor state becomes unstable and the tuned state starts moving around the ring. For constant external inputs, the system moves eventually at a constant speed according to $\psi_{A}=v t / \tau$, where $v$ is the angular velocity in units of $\tau$. The equations governing the system are

$$
\begin{aligned}
s_{A 0}= & I_{A 1} f_{0}\left(\theta_{A}\right), \quad A=e, l, r \\
s_{A 1}= & I_{A 1} f_{1}\left(\theta_{A}\right) \frac{1}{\sqrt{1+v^{2}}}, \quad A=e, l, r \\
\theta_{A}= & \arccos \left(-\frac{I_{A 0}}{I_{A 1}}\right), \quad A=e, l, r \\
0= & (\sin \alpha-v \cos \alpha) s_{r 1}-(\sin \alpha+v \cos \alpha) s_{l 1} \\
I_{e 0}= & I-\frac{K_{0}}{2}\left(s_{l 0}+s_{r 0}\right) \\
I_{l 0}= & I_{l}+H_{0} s_{e 0}-L_{0} s_{r 0} \\
I_{r 0}= & I_{r}+H_{0} s_{e 0}-L_{0} s_{l 0} \\
I_{e 1}= & \frac{K_{1}}{2 \sqrt{1+v^{2}}}\left[(\cos \alpha-v \sin \alpha) s_{l 1}\right. \\
& \left.+(\cos \alpha+v \sin \alpha) s_{r 1}\right] \\
I_{A 1}= & H_{1} s_{e 1}, \quad A=l, r
\end{aligned}
$$

These equations provide the angular velocity $v$ of the traveling bump as a function of the difference between the external inputs $I_{l}$ and $I_{r}$. In particular, the speed is given by

$$
v=\frac{1}{\rho}\left(\sqrt{1+\rho^{2}}-1\right)
$$

where

$$
\begin{aligned}
\rho & =\frac{\tan \alpha\left|s_{l 1}-s_{r 1}\right|}{s_{l 1}+s_{r 1}} \\
& =\frac{\tan \alpha\left|f_{1}\left(\theta_{l}\right)-f_{1}\left(\theta_{r}\right)\right|}{f_{1}\left(\theta_{l}\right)+f_{1}\left(\theta_{r}\right)}
\end{aligned}
$$

For a small difference $\delta I \equiv I_{l}-I_{r}$ we obtain, for $L_{0}<\pi / \theta_{i}$ :

$$
v=\frac{\tan \alpha \theta_{i}}{4 f_{1}\left(\theta_{i}\right) H_{1} I_{e 1} f_{1}\left(\theta_{e}\right)\left(\pi-L_{0} \theta_{i}\right)} \delta I
$$

For $L_{0}>\pi / \theta_{i}$, any $\delta I$ leads to a finite speed. The stationary bump is unstable even for equal inputs to left and right DTNs.
When $\delta I$ goes beyond a critical value, one of the two inhibitory populations, e.g. $l$ if $I_{l} \gg I_{r}$, becomes silent. At this point, we obtain from Eq. (68) that the velocity becomes independent from the difference between the two external inputs: $\rho=\tan \alpha$, and consequently

$$
v=\frac{1-\cos \alpha}{\sin \alpha}
$$

is the saturation velocity of the network. Equation (71) shows that the saturation velocity depends only on the offset $\alpha$ in the connections from the inhibitory to the excitatory population. It increases with $\alpha$, as $\alpha / 2$ for $\alpha$ small, up to 1 when $\alpha$ tends towards $\pi / 2$. For instance, for time constants $\tau$ of the order of $10 \mathrm{~ms}$, this means a saturation velocity that can reach up to $5000^{\circ} / \mathrm{s}$. This velocity is much larger than typical angular velocities experienced by a rat in natural conditions. Figure 5 shows the saturation velocity as a function of $\alpha$.

The velocity of the traveling bump is shown as a function of the differential input $\delta I$ for different values of $\alpha, L_{0}$ and $K$ in Fig. 6 . Note that the curves (velocity vs differential input) can be far from linear in the sub-saturation range. In particular, for low values of $L_{0}\left(L_{0}=0.5\right.$ in Fig. 6), the curves are linear only in a very small range of inputs. On the other hand, for intermediate values of $L_{0}$, the curves can be linear in a large range (see for example the curves, corresponding to $\alpha=75$ and $\alpha=85^{\circ}$ respectively in the panel $L_{0}=1$ ). The range where linearity holds is optimal at intermediate values of $\alpha,\left(\alpha\right.$ about $75^{\circ}$ for $L_{0}=1, K=1.5 K_{c}$, where $K_{c}=4 /\left(H_{1} \cos \alpha\right)$ is the value at which the bump state appears). Finally, increasing $L_{0}$ too much

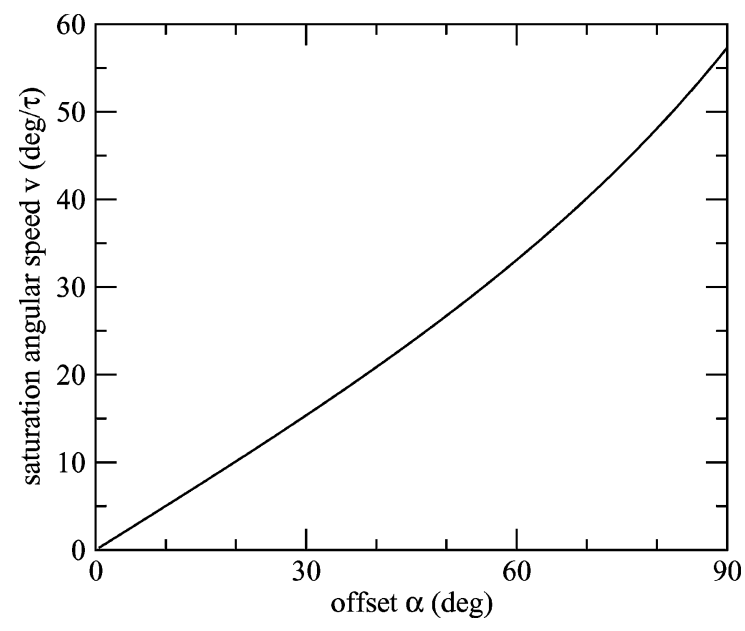

Figure 5. Saturation angular velocity as a function of the offset $\alpha$. 

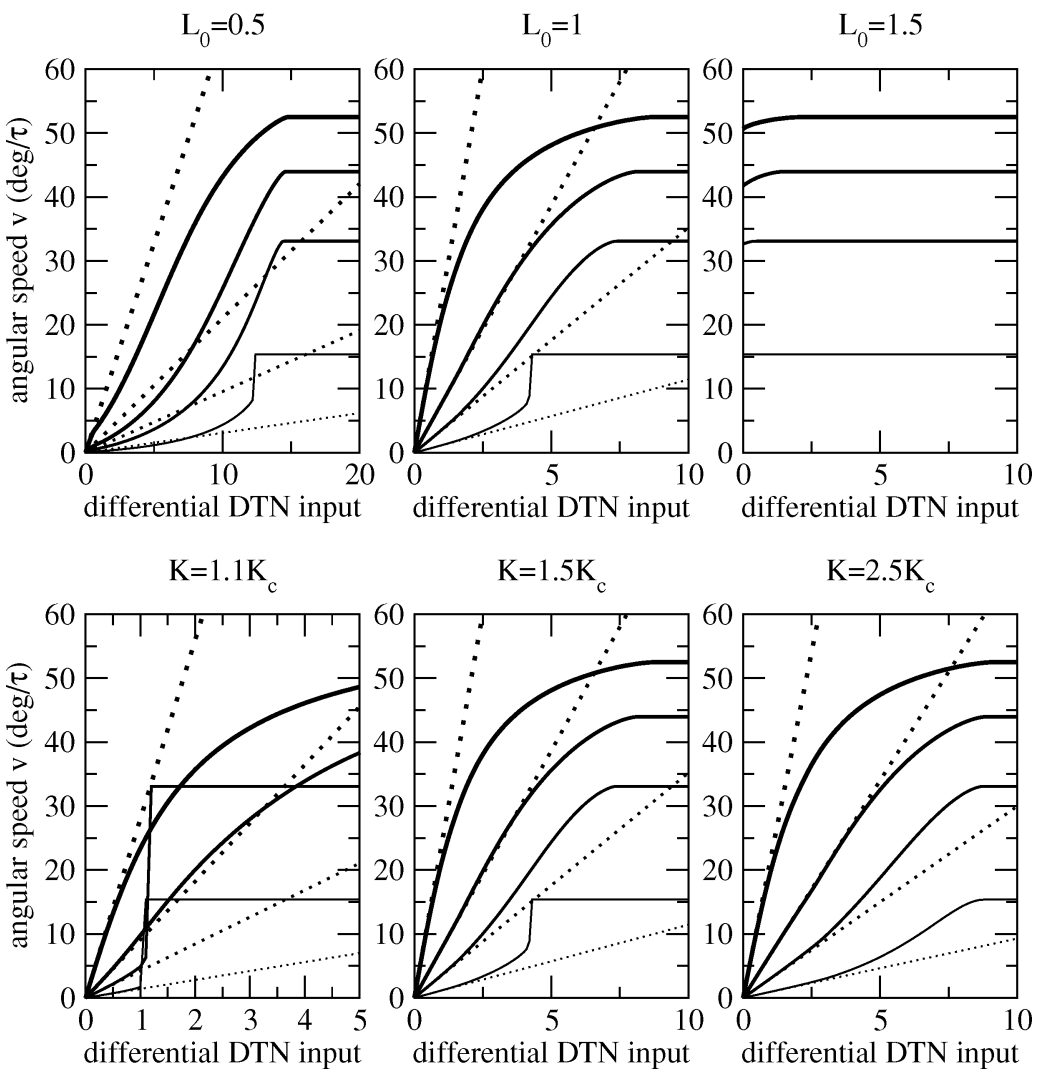

Figure 6. Angular velocity of the bump vs differential input $\delta I$ for different values of $\alpha, L_{0}$, and $K$. Each panel displays four sets of curves corresponding to four values of $\alpha$ (from thickest line to thinnest line, $\alpha=85^{\circ}, 75^{\circ}, 60^{\circ}, 30^{\circ}$; solid curves, velocity vs $\delta I$ as obtained from solving Eqs. (58)-(66) numerically; dashed curves, slope at the origin, as obtained from Eq. (70)). Top panels: $K_{0}=K_{1}=K=6 H_{1} / \cos (\alpha), L_{0}$ is varied (value indicated on top of each panel). Bottom panels: $L_{0}=1, K_{0}=K_{1}=K$ is varied $\left(K_{c}=4 H_{1} / \cos (\alpha)\right.$ is the critical value of the coupling beyond which the tuned states appear). Other parameters: $H_{0}=H_{1}=1+L_{0}, I_{I}=0$, and $I_{E}=10(1+K)$.

causes a loss of stability of the stationary bump state. Hence, the bump moves at a finite speed even if there is no differential input to the DTN networks. Finally, the range of linearity tends to increase as the networks become more sharply tuned, as shown by the bottom panels of Fig. 6 where $K$ is increased from $1.1 K_{c}$ to $2.5 K_{c}$.

\section{An Attractor-Integrator Network of Spiking Neurons}

This section presents simulations of a network of spiking neurons with the architecture of Fig. 1. The model simulated in this section differs in many respects from the one of Section 3: neurons are spiking leaky integrate-and-fire neurons rather than analog firing rate units; footprints have a Gaussian shape rather than cosine; synaptic currents mimic the time courses of AMPA, NMDA, and GABA receptors. Nonetheless, these simulations allow us to show that the main results obtained in Section 3 hold qualitatively in this more realistic network.

\subsection{Methods}

4.1.1. Model Architecture. The architecture of the attractor-integrator network is as defined in Section 3 and shown in Fig. 1. The LMN network is implemented as a population of $N_{E}=1000$ excitatory directional neurons, and $\mathrm{DTN}_{l, r}$ are two networks of $N_{I}=1000$ inhibitory directional units. The preferred directions $\theta_{e, l, r}$ are evenly distributed over all possible directions.

All LMN neurons receive an external excitatory input $I_{E}$ inducing a mean background activity of $80 \mathrm{~Hz}$. LMN receives also inhibitory afferents from $\mathrm{DTN}_{l}$ and $\mathrm{DTN}_{r}$. The projections from cells in 
$\mathrm{DTN}_{l, r}$ with preferred directions $\theta_{l, r}$ to a cell in LMN with label $\theta_{e}$ have a weight $K\left(\theta_{e}-\theta_{l, r}-\pi \pm \alpha\right)$ with a Gaussian profile as a function of the distance between $\theta_{e}$ and $\theta_{l, r}$, where the constant $\alpha=50^{\circ}$ indicates an angular offset. For instance, the strength of the projection $l \rightarrow e$ is proportional to

$$
K_{e l}\left(\theta_{e}-\theta_{l}\right)=K_{1} \exp \left(-\left(\theta_{e}-\theta_{l}-\pi+\alpha\right)^{2} / 2 \sigma_{I}^{2}\right)
$$

where $K_{1}=1.1$ and $\sigma_{I}=30^{\circ}$ define, respectively, the amplitude and the width of the synaptic weight function. Thus, a unit in $\mathrm{DTN}_{l}$ with preferred direction $\theta_{l}$ inhibits mostly the neuron in LMN with preferred direction $\theta_{e}=\theta_{l}+\pi-\alpha$. Conversely, a cell in DTN $_{r}$ with label $\theta_{r}$ sends the strongest inhibitory signal to the neuron in LMN with preferred direction $\theta_{e}=\theta_{r}+\pi+\alpha$.

The networks $\mathrm{DTN}_{l, r}$ receive afferent excitation from LMN by means of iso-directional projections. The synaptic efficacy of the projection from a neuron in LMN with label $\theta_{e}$ to a neuron in $\mathrm{DTN}_{l, r}$ is given by $H\left(\theta_{e}-\theta_{l, r}\right)$, where $H$ is a gaussian weight function centered at $\theta_{l, r}$, with amplitude $H_{1}=0.3$ and width $\sigma_{E}=80^{\circ}$. As described in the next section, the excitatory connections from LMN to DTN, $H\left(\theta_{e}-\theta_{l, r}\right)$, are mediated by AMPA and NMDA receptors. Note that the Gaussian kernels that define how LMN and DTN networks are wired (Eq. (72)) allow us to obtain Gaussian shaped tuning curves which are close to those observed in rat HD cells (see simulation results in Section 4.2.1).

The two populations $\mathrm{DTN}_{l, r}$ are also fully interconnected by means of reciprocal inhibitory projections of strength $L_{0}=0.02$. Finally, each unit in $\mathrm{DTN}_{l, r}$ receives an external excitatory input current $I_{I}+I_{l, r}\left(v_{h}\right)$, where $I_{I}$ is a background input, while $I_{l, r}\left(v_{h}\right)$ is proportional to the amplitude of the head angular velocity $v_{h}(t)$. In particular, the input current $I_{r}\left(v_{h}\right)$ signals clockwise head rotations and is taken as $I_{r} \propto\left|v_{h}\right|$ for $v_{h}<0, I_{r}=0$ otherwise. On the other hand, $I_{l} \propto\left|v_{h}\right|$ for $v_{h}>0, I_{l}=0$ otherwise, signals counterclockwise head turns.

4.1.2. Neuron and Synapse Models. The basic components of the model are: (i) leaky integrate-and-fire spiking neurons, (ii) synapses mediated by AMPA and NMDA receptors (for the excitatory synapses) and by GABA receptors (for the inhibitory synapses). The de- scription of the dynamics of the neurons and of the synaptic transmission is similar to Brunel and Wang (2001) and can be considered as a generalization of Eqs. (11)-(16) for spiking units.

Let $V_{\text {leak }}, V_{\text {threshold }}$, and $V_{\text {reset }}$ denote the resting membrane potential, the firing threshold, and the reset potential of a neuron, respectively. Let $I(t)$ be the total synaptic drive received by a cell from other units. The dynamics of the membrane potential $V(t)$ is given by

$$
C \cdot \frac{d V(t)}{d t}=-g \cdot\left(V(t)-V_{\text {leak }}\right)-I(t)
$$

where $C$ and $g$ are, respectively, the membrane capacitance and leak conductance $(\tau=C / g$ is the membrane time constant). We take $V_{\text {leak }}=-70 \mathrm{mV}, V_{\text {threshold }}=$ $-50 \mathrm{mV}$, and $V_{\text {reset }}=-55 \mathrm{mV}$ for all neurons of the model. For LMN units the membrane capacitance $C_{e}$ is $0.5 \mathrm{nF}$, and the conductance $g_{e}$ is $25 \mathrm{nS}$. Thus, the membrane time constant is $\tau_{e}=C_{e} / g_{e}=20 \mathrm{~ms}$. For DTN $\mathrm{D}_{l, r}$ units, $C_{l, r}=0.2 \mathrm{nF}, g_{l, r}=20 \mathrm{nS}$, and $\tau_{l, r}=10 \mathrm{~ms}$. Whenever the membrane potential $V(t)$ reaches the threshold $V_{\text {threshold }}$ the neuron emits one spike. Then, its membrane potential is reset to $V_{\text {reset }}$ and the dynamics of $V(t)$ is frozen during a refractory period $t_{\text {ref }}$. We take $t_{\text {ref }}=2 \mathrm{~ms}$ for LMN units and $t_{\text {ref }}=1 \mathrm{~ms}$ for $\mathrm{DTN}_{l, r}$ units.

The total synaptic input to a $\mathrm{LMN}$ neuron is

$$
I(t)=I_{E}+I_{\mathrm{GABA}}(t)
$$

whereas for a DTN neuron it is given by

$$
I(t)=I_{I}+I_{l, r}(t)+I_{\mathrm{AMPA}}(t)+I_{\mathrm{NMDA}}(t)+I_{\mathrm{GABA}}(t)
$$

The inhibitory synaptic input $I_{\mathrm{GABA}}(t)$ is defined as

$I_{\mathrm{GABA}}(t)=g_{\mathrm{GABA}} \cdot\left(V(t)-V_{I}\right) \cdot \sum_{j=0}^{n_{\mathrm{GABA}}} s_{j}^{\mathrm{GABA}}(t)$

where $g_{\mathrm{GABA}}=1.25 \mathrm{nS}, V_{I}=-70 \mathrm{mV}$, and $n_{\mathrm{GABA}}$ is the number of GABAergic afferents $\left(2 N_{I}\right.$ for LMN cells, $N_{I}$ for DTN cells). The term $s_{j}^{\mathrm{GABA}}(t)$ is a gating variable (fraction of open channels) of the $j$ th GABAergic synapse, whose dynamics is defined by Eq. (79).

The AMPA-mediated contribution to the total synaptic input is given by

$$
I_{\mathrm{AMPA}}(t)=g_{\mathrm{AMPA}} \cdot\left(V(t)-V_{E}\right) \cdot \sum_{j=0}^{n^{\mathrm{AMPA}}} s_{j}^{\mathrm{AMPA}}(t)
$$


where $g_{\mathrm{AMPA}}=0.016 \mathrm{nS}, V_{E}=0 \mathrm{mV}$, and $s_{j}^{\mathrm{AMPA}}(t)$ is the gating variable.

The synaptic drive due to the NMDA receptors is

$$
\begin{aligned}
I_{\mathrm{NMDA}}(t)= & \frac{g_{\mathrm{NMDA}} \cdot\left(V(t)-V_{E}\right)}{1+\left[\mathrm{Mg}^{2+}\right] \exp (-0.062 V(t)) / 3.57} \\
& \cdot \sum_{j=0}^{n^{\mathrm{NMDA}}} s_{j}^{\mathrm{NMDA}}(t)
\end{aligned}
$$

where $g_{\mathrm{NMDA}}=0.258 \mathrm{nS}, s_{j}^{\mathrm{NMDA}}(t)$ is the gating variable, and the voltage dependence of the conductance is taken from Jahr and Stevens (1990), assuming an extracellular concentration of magnesium $\left[\mathrm{Mg}^{2+}\right]=$ $1 \mathrm{mM}$.

The gating variables $s_{j}(t)$ are defined according to the following equation

$$
\frac{d s_{j}(t)}{d t}=-\frac{s_{j}(t)}{\tau_{\text {decay }}}+\sum_{k} \delta\left(t-t_{j}^{k}\right) \cdot w_{j}
$$

where the decay time constant $\tau_{\text {decay }}$ is $5 \mathrm{~ms}$ for GABA receptors, $2 \mathrm{~ms}$ for AMPA receptors, and $100 \mathrm{~ms}$ for NMDA receptors. The term $\sum_{k} \delta\left(t-t_{j}^{k}\right) \cdot w_{j}$ is a weighted sum over the spikes emitted by the presynaptic neuron $j$, modeled as delta functions. The weight $w_{j}$ is equal to $K_{e, l}\left(\theta_{e}-\theta_{l}\right)$ for the inhibitory projections from DTN to LMN, it is equal to $L_{0}$ for the reciprocal DTN inhibitory connections, and it is equal to $H\left(\theta_{e}-\theta_{l, r}\right)$ for the excitatory projections from LMN to DTN.

4.1.3. External Inputs. Each neuron receives extrinsic background noise simulating external spontaneous activity (corresponding to $I_{E}$ for LMN units and to $I_{I}$ for DTN). This noise consists of Poisson spike trains transmitted through AMPA synaptic receptors with conductances $g_{E}^{\mathrm{AMPA}}=2.08 \mathrm{nS}$ and $g_{I}^{\mathrm{AMPA}}=1.62 \mathrm{nS}$. The frequency of the spike train is such that, in the absence of recurrent inputs, DTN neurons are below threshold for firing, whereas LMN neurons fire, in average, at about $80 \mathrm{~Hz}$. This background LMN activity, along with other free parameters of the model, was set to obtain a mean peak firing rate of LMN HD cells of about $60 \mathrm{~Hz}$ in the presence of recurrent inhibitory inputs (see simulation results in Section 4.2.1).

4.1.4. Choice of Parameters. Neuronal and synaptic parameters have been taken to be similar to previous modeling studies (e.g., Brunel and Wang, 2001) and are consistent with corresponding experimental measurements. On the other hand, the parameters defining the architecture (synaptic footprints, external inputs) are not constrained by current experimental data. The values of these parameters were set such that the tuning curves in both DTN and LMN are consistent with experimental data. The values of the offset $\alpha$ and the mutual inhibitory projections $L_{0}$ can be set to obtain a good performance of the model in terms of integration of angular velocity signals (see below). The balance between NMDA and AMPA conductances was set in order to get stable asynchronous activity. When the post-synaptic membrane potential is at the resting level, the resulting ratio between the NMDA and AMPA contribution to a single excitatory post-synaptic potential (EPSP) is about 0.5 in terms of the peak of the EPSP, and about 20 in terms of the integral of the EPSP over time.

4.1.5. Population Vector Coding. In the model, the ongoing animal's heading is encoded by means of a bell-shaped activity profile distributed over the LMN HD cell population. In order to reconstruct the current head direction $\theta(t)$ in bins $[t, t+\Delta t]$, a population vector scheme (Georgopoulos et al., 1986) is employed to decode the ensemble HD cell activity:

$$
\bar{\theta}(t)=\arctan \left(\frac{\sum_{e=1}^{N_{E}} \sin \left(\theta_{e}\right) n_{e}(t, t+\Delta t)}{\sum_{e=1}^{N_{E}} \cos \left(\theta_{e}\right) n_{e}(t, t+\Delta t)}\right)
$$

where $\theta_{e}$ is the preferred direction of unit $e$, and the function $n_{e}(t, t+\Delta t)$ is equal to 1 if the neuron $e$ fires in the interval $[t, t+\Delta t], 0$ otherwise.

\subsection{Simulation Results}

4.2.1. Stationary Attractor State Regime. In this section we take $I_{l}=I_{r}=0$ to simulate the situation in which the head of the animal remains still (zero head angular velocity) over time. Therefore, the two networks $\mathrm{DTN}_{l, r}$ do not receive any differential modulatory signal and the dynamics of the LMN-DTN system depends upon the intrinsic excitatory-inhibitory loop.

4.2.1.1. Persistence and Stability of the HD Signal. We first checked that the tuned state emerges from random initial configurations for the parameter set given in Section 4.1. For this parameter set, the uniform state is unstable and, after a transient period of about $200 \mathrm{~ms}$, a tuned attractor state emerges: a sub-group of LMN units with similar preferred directions discharge tonically, 
whereas the others exhibit a very low baseline frequency. Such a self-sustained attractor state provides a stable directional coding over a scale of seconds. To assess the stability of the self-sustained bump of activity we run a series of $n$ simulations, where in each trial $1 \leq i \leq n$ we measure the average center of mass of the activity profile $\bar{\theta}_{i}$ and the standard deviation $\sigma_{i}$ of the temporal fluctuations around $\bar{\theta}_{i}$, in the interval of $15 \mathrm{~s}$ duration. The mean standard deviation, calculated over $n=40$ trials, is $\sigma=19.1^{\circ}$.

For the 'standard' set of parameters, the tuning curve of LMN units has a mean peak firing rate of 60-65 spikes/s and a directional firing range of about $180^{\circ}$ (Fig. 7A). DTN units have a mean peak firing rate of about 25 spikes/s and a directional firing range of about $250^{\circ}$ (Fig. 7B). These values are consistent with those

A

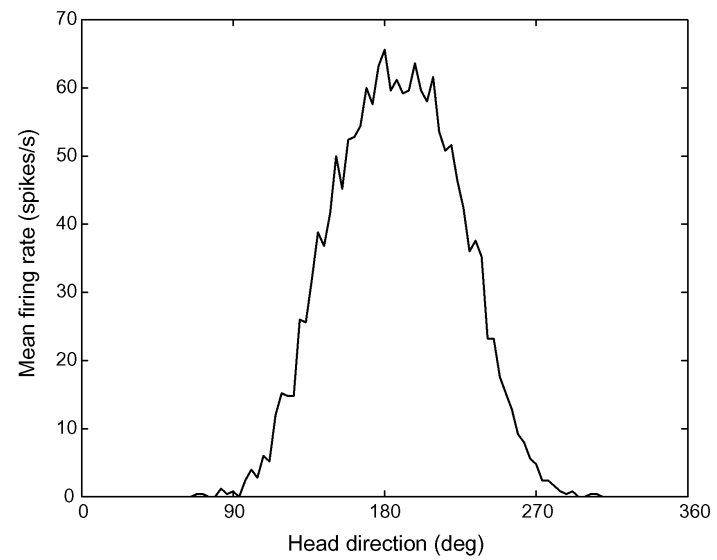

B

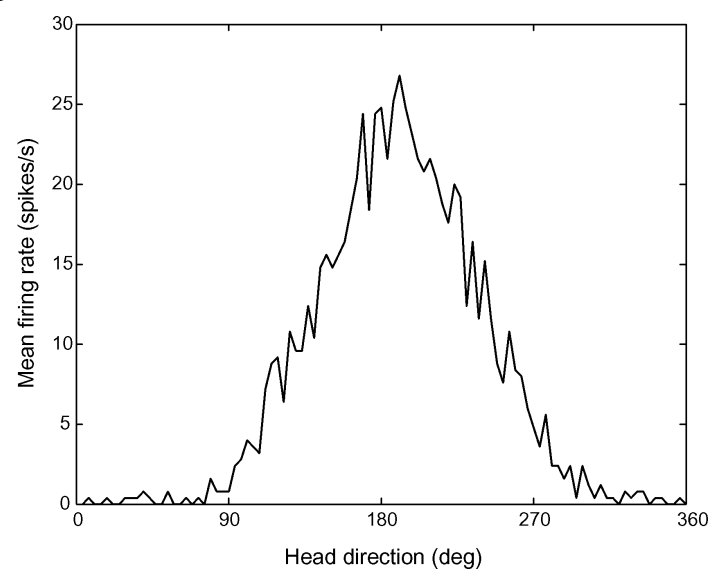

Figure 7. Tuning curves of the formal HD cells in LMN (A) and DTN (B). The mean peak spike frequency is about 60-65 spikes/s for LMN units and 25 spikes/s for DTN cells. The width of the gaussian activity profile is $180^{\circ}$ for LMN and $250^{\circ}$ for DTN. observed experimentally when recording HD cells in the rat LMN and DTN (Taube, 1998; Bassett and Taube, 2001b).

One of the main predictions of the analysis, Eqs. (43)-(49), is that a tuned state emerges when the parameter $H_{1}$ (the maximum weight of the excitatory projections from LMN to DTN) and/or the parameter $K_{1}$ (the maximum weight of the inhibitory projections from DTN to LMN) are strong enough. In the threshold-linear network, the condition for a tuned state to emerge is given by $H_{1} K_{1} \cos \alpha>4$, where $\alpha$ is the angular offset of the connections from DTN to LMN. To check that a qualitatively similar condition holds in the network of integrate-and-fire neurons, we performed a series of simulations keeping the angular offset $\alpha$ fixed and multiplying both parameters $K_{1}$ and $H_{1}$ of the 'standard' set of parameters by a single parameter $0 \leq m \leq 2$. For each $m$, we let the network evolve during $1 \mathrm{~s}$. Consistent with the analytical result, we found that there exists a threshold value $m \approx 0.6$ above which a gaussian-shaped attractor state emerges from a uniform initial condition.

\subsubsection{Influence of the Mutual Inhibitory Projections} of the two DTN Networks on the Stability of the Head Direction Signal. We studied the stability of the tuned state as a function of the strength $L_{0}$ between the two inhibitory populations $\mathrm{DTN}_{l, r}$. In our 'standard' set of parameters, Section 4.1.1, $L_{0}=0.02$. Here $L_{0}$ is varied in the range $[0.03,0.15]$. For each value $L_{0}$ we let the network evolve during $5 \mathrm{~s}$. Then we compute both the mean angular velocity $v$ of the bump of activity in LMN and the variance around $v$ over the $5 \mathrm{~s}$ period.

Figure 8A shows the values of the mean angular velocity $v$ (solid curve) and its variance (dashed curve) as a function of $L_{0}$, averaged over $n=5$ trials. Interestingly, the strength of the mutual inhibition between the two DTN networks plays an important role in determining the stability of the tuned attractor state. For $L_{0}<0.06$, the network exhibits an equilibrium regime in which the bump of activity remains stable over time (small angular speed and small variance over $5 \mathrm{~s}$ ). For $L_{0}>0.06$, the HD attractor state becomes unstable and enters a transitory regime showing an irregular behavior (the angular speed increases and the variance over $5 \mathrm{~s}$ becomes very large). For $L_{0}>0.07$, the network dynamics is characterized by a new regime in which the tuned state moves around the ring attractor with a constant angular velocity, and the variance is again very small. 
A

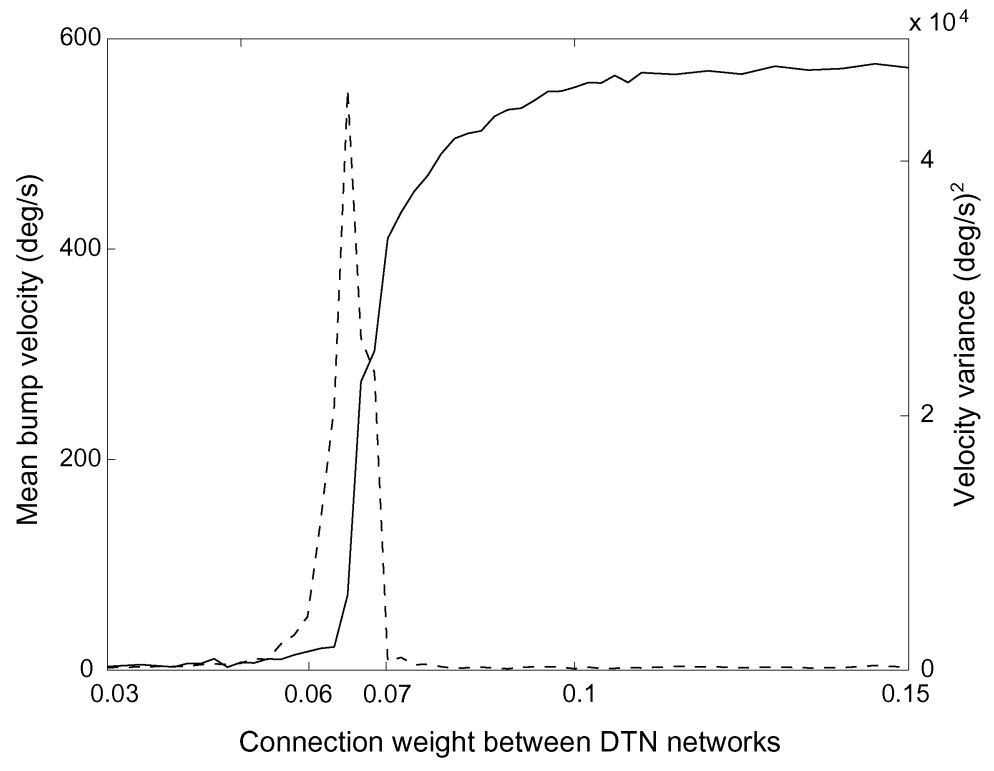

B
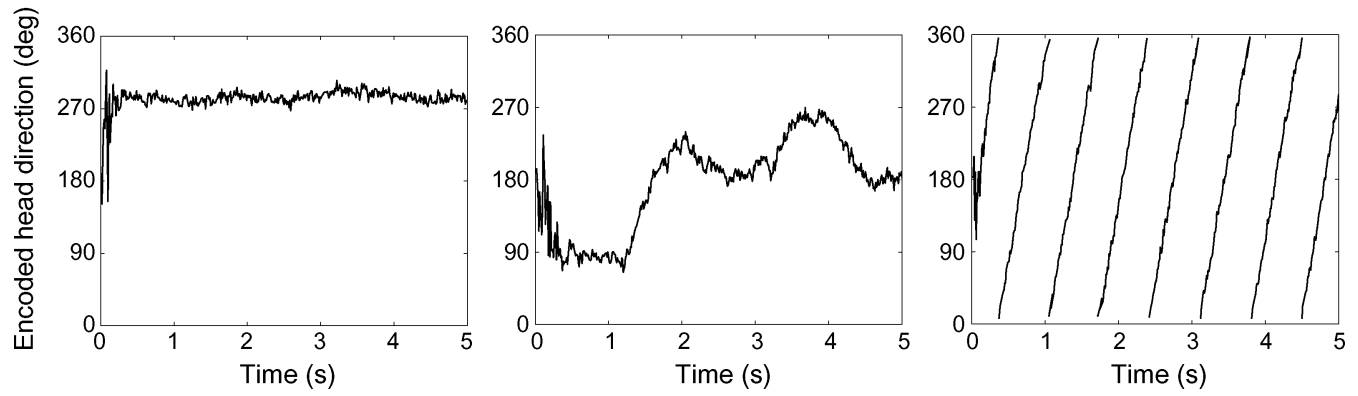

Figure 8. Stability of the tuned state as a function of the strength $L_{0}$ of the mutual inhibitory connections between the two DTNs. (A) For each value $0.03 \leq L_{0} \leq 0.15$ we measure the angular velocity $v$ (solid curve) of the bump of activity in LMN and the variance around $v$ over a $5 \mathrm{~s}$ period (dashed curve). For $L_{0}<0.06$, the tuned attractor state remains stable over time (angular speed $v$ close to zero). Around $L_{0}>0.06$, the stationary bump becomes unstable, and the tuned state starts moving around the ring. Close to the bifurcation, there are large fluctuations of the angular velocity: the bump moves in a very irregular fashion ( $v$ increases and the variance is very large). For $L_{0}>0.07$ the tuned state moves around the ring with a constant angular velocity $v$, with small fluctuations around $v$. (B) Encoded head direction $\bar{\theta}(t)$ over a $5 \mathrm{~s}$ trial for three particular values of $L_{0}$. The curves represent the center of mass of the ensemble LMN activity computed according to Eq. (80). Left: for $L_{0}=0.03$ the bump of activity remains stable over time. Center: for $L_{0}=0.062$ the attractor state exhibits a random angular velocity profile. Right: for $L_{0}=0.09$ the bump moves at constant angular velocity.

Figure 8B shows three samples of network dynamics given three specific values of the inhibitory coupling $L_{0}$. Each diagram displays the center of mass of the ensemble LMN activity (Eq. (80)) over time. As previously mentioned, with our set of parameters about $200 \mathrm{~ms}$ are necessary for a tuned attractor state to emerge. For $L_{0}=0.03$ (left panel), a balance between the two DTN $\mathrm{N}_{l, r}$ occurs and the bump of activity remains stable (small mean angular velocity $v$ and small variance around $v$ ). For $L_{0}=0.09$ (right panel), one of the two DTNs dominates over the other and the tuned state travels around the ring at constant velocity (small variance). For $L_{0}=0.062$ (central diagram), the stochastic nature of the external excitatory drive (Poisson noise, Section 4.1.2) makes the two DTN dominate each other alternately. This results in abrupt random changes, in both amplitude and sign, of the bump angular velocity, and yields a large value of the variance (see the peak in the variance vs $L_{0}$ curve in Fig. 8A). 
4.2.2. Integration of the Head Angular Velocity. For non-zero head angular velocity, the two $\mathrm{DTN}_{l, r}$ networks receive a differential external modulation, i.e. $I_{l} \neq I_{r}$. This generates an asymmetry within the LMNDTN coupling and yields a stronger inhibition at one side of the LMN bump of activity. As a consequence, the 'bump' state becomes unstable and starts moving.

\subsubsection{Response of the System to an External Constant} Angular Velocity Signal. Figure 9 (circle data points) shows the response of the system to a set of constant head angular velocities corresponding to counterclockwise rotations, i.e. $I_{l}>0$ and $I_{r}=0$. The continuous angular velocity space is discretized by taking bins of $20 \%$ s. Each angular velocity is applied to the HD cell system for a period of $4 \mathrm{~s}$, and the mean angular velocity of the bump state induced by the external input $I_{l}$ is measured. The figure, obtained by averaging over $n=5$ experiments, shows that the system integrates angular velocities within the range $0<v_{h}<400^{\circ} / \mathrm{s}$ properly. Above $400 \%$ s the response of the system becomes non-linear and, as predicted by the analytical model in Section 3.2, for $I_{l} \gg I_{r}$ a saturation of the bump angular velocity occurs. The saturation velocity

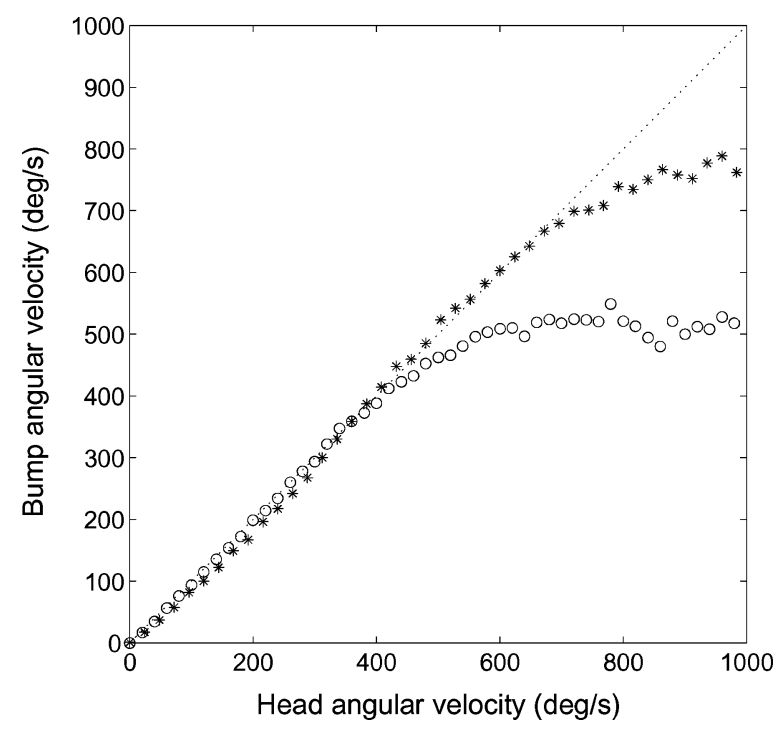

Figure 9. For non-stationary regimes, e.g. $I_{l}>0$ and $I_{r}=0$, the tuned state shifts around the ring with a speed proportional to the angular velocity encoded by the differential input $\delta I(t)$, e.g. $\delta I(t)=$ $I_{l}(t)-I_{r}(t)$. The two sets of symbols show the angular velocity of the tuned state (averaged over $4 \mathrm{~s}$ ) as a function of constant head angular velocity, for two values of the angular offset in the projections from DTN $_{l, r}$ to LMN (circles: $\alpha=50^{\circ}$, saturation occurs $v$ at $\approx 520^{\circ} / \mathrm{s}$; stars: $\alpha=65^{\circ}$, the saturation velocity increases to $v \approx 780^{\circ} / \mathrm{s}$ ). is about $520 \%$ s. Note that this value is of the same order of magnitude as the analytical estimate of Eq. (71), taking as $\tau$ the longest time constant of the system, which is that of NMDA receptors of about $100 \mathrm{~ms}$.

The analysis of the previous section predicts that the saturation velocity depends only on the angular offset $\alpha$ in the projections from $\mathrm{DTN}_{l, r}$ to $\mathrm{LMN}$ (Eq. (71)). In our standard set of parameters, $\alpha=50^{\circ}$. We run another series of simulations to assess the response of the system to a set of constant angular velocities when taking a larger angular offset $\alpha^{\prime}=65^{\circ}$. The star data points in Fig. 9 show the results averaged over $n=$ 5 trials. Consistent with the analytical prediction, the system can integrate angular velocities within a larger velocity range $[0,700 \%$ s and the saturation velocity is about $780^{\circ} \%$.

4.2.2.2. Tracking a Rat Angular Velocity Profile. To test the integration property of the system on actual recorded data, we apply a set of $n=35$ rat head angular velocity profiles during a period of $120 \mathrm{~s}$. These angular velocity profiles were recorded by sampling at $60 \mathrm{~Hz}$ the heading of four Long-Evans rats freely moving on a small circular platform $20 \mathrm{~cm}$ in diameter (Arleo, Zugaro, Déjean, Wiener, unpublished observations). Figure 10 shows one example of the heading $\bar{\theta}(t)$ (gray line) encoded by the LMN-DTN attractor-integrator when tracking the rat's orientation

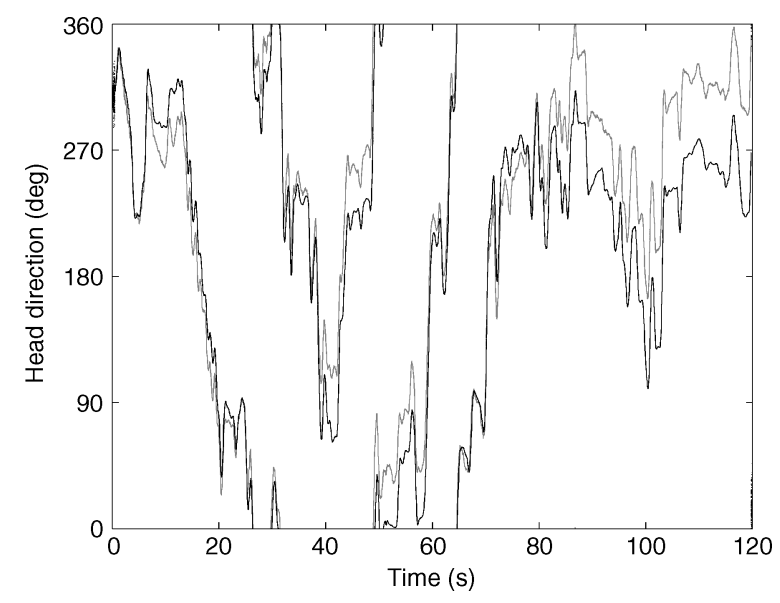

Figure 10. The angular velocity profile $v(t)$ of a freely moving rat is applied to the HD system for $120 \mathrm{~s}$. The model integrates $v(t)$ over time providing an ongoing estimate $\bar{\theta}(t)$ (gray line) of the rat's heading $\theta(t)$ (black line). One example out of $n=35$ simulations is shown here (for sake of clarity, the two curves have been smoothed by means of an averaging time window of $500 \mathrm{~ms}$ ). 


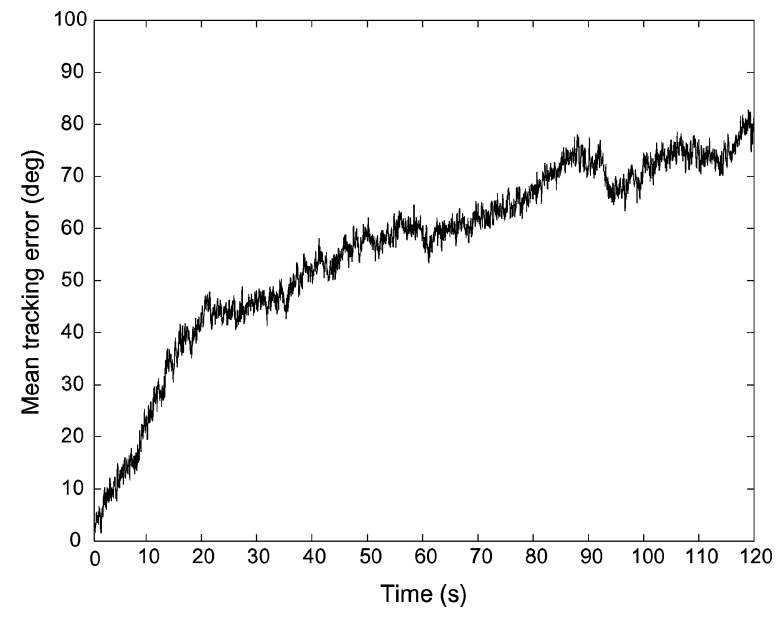

Figure 11. Mean reconstruction error, averaged over $n=35$ trials, of the HD system when tracking angular velocity profiles of freely moving rats during $120 \mathrm{~s}$. The diagram shows that the integration of head angular movements based on inertial signals only is prone to cumulative drift over time.

$\theta(t)$ (black line) over time. During the first part of the simulation, the HD network provides an appropriate estimate of $\theta(t)$. Then, since the integration of angular displacements based on inertial self-motion signals is prone to cumulative drift (Matthews et al., 1988; McNaughton et al., 1991; Goodridge et al., 1998), the system fails to maintain a stable directional coding over time. To quantify the tracking performance of the HD system we compute the mean reconstruction error $\epsilon(t)=1 / n \sum_{i=1}^{n}\left|\bar{\theta}_{i}(t)-\theta_{i}(t)\right|$, with $\epsilon \in\left[0^{\circ}, 180^{\circ}\right]$. Figure 11 displays the mean tracking error function $\epsilon(t)$ obtained by averaging over the $n=35$ trials. Consistent with earlier hypotheses (Taube et al., 1990b; McNaughton et al., 1991; Skaggs et al., 1995; Knierim et al., 1998; Arleo and Gerstner, 2001), our system predicts that incorporating allothetic sensory inputs (e.g., vision, touch) is necessary to recalibrate pure inertial signals and achieve robust directional coding.

\subsubsection{Response of the HD Cell System to Reorienting}

External Inputs. Electrophysiological data demonstrate that HD cells are strongly influenced by salient visual cues of the environment (Taube et al., 1990b; McNaughton et al., 1991; Goodridge and Taube, 1995; Knierim et al., 1998; Zugaro et al., 2001). For instance, rotating a familiar visual landmark by an angle $\beta$ induces a rotation $\beta^{\prime} \approx \beta$ of the preferred directions of all HD cells. Recently, Zugaro et al. (2003) have quantitatively measured the time necessary for the preferred directions of HD cells in the rat ADN to be updated by a reorienting visual cue. The experimental setup consisted of a black high-walled cylinder with a large white card attached to the inner wall which provided the dominant visual landmark. The preferred directions of the HD cells were first measured in light conditions. Then, in the dark, the cue card was rotated by an angle $\beta=$ $90^{\circ}$. Finally, the light was switched back on, which triggered the reorientation $\beta^{\prime}$ of the preferred directions. The authors observed a mean latency of $80 \pm 10 \mathrm{~ms}$ for a HD cell to go from baseline to peak firing activity (i.e., establishment of a new bump following the reorientation event). On the other hand, they found that $140 \pm 10 \mathrm{~ms}$ were necessary for a cell to return to baseline activity from its peak firing rate (i.e., extinction of the bump existing prior the reorientation event).

In this section we focus on the temporal aspects of the state transition in our HD cell model. To simulate a reorientation event, we apply a strong external stimulus $R$ to the LMN network at time $t_{R}=1 \mathrm{~s}$. In the simulation, the stimulus is conveyed to LMN neurons by modulating the external current $I_{E}$ that generates the background spontaneous activity (Section 4.1.2). In particular, for each LMN cell $i$, we modulate the conductance $g_{E}^{\text {AMPA }}$ by adding a gaussian component according to:

$$
\begin{aligned}
g_{E}^{\mathrm{AMPA}^{\prime}}(t)=g_{E}^{\mathrm{AMPA}} & (1+R(t) \\
& \left.\cdot \exp \left(-\left(\theta_{R}-\theta_{i}\right)^{2} / 2 \sigma_{R}^{2}\right)\right)
\end{aligned}
$$

where $\theta_{R}$ is the allocentric direction of the reorienting stimulus, $\theta_{i}$ denotes the preferred direction of cell $i$, and $\sigma_{R}=45^{\circ}$ is the width of the gaussian profile. The amplitude of the stimulus decreases exponentially over time according to the term $R(t)=R_{0} \exp (-t / \tau)$, for $t>t_{R} . R_{0}=1.3$ is chosen such that a new bump appears close to $\theta_{R}$ and destabilizes the previous bump. An exponential decay with $\tau=100 \mathrm{~ms}$ was used to prevent the firing rates in LMN and DTN to become very high.

With such parameters of the input, the dynamics of the system rates following reorientation matched qualitatively the experimental data of Zugaro et al. (2003), as shown in Fig. 12. A new bump appears at the location of the reorienting stimulus after a few tens of ms. The appearance of this bump in turn switches the old bump, due to an overall increase in DTN inhibition. The old bump decays in about $100 \mathrm{~ms}$. To compare the simulation data with the experimental data, we normalized between 0 and 1 the instantaneous firing rates (computed in $10 \mathrm{~ms}$ bins) of two populations of cells: those 

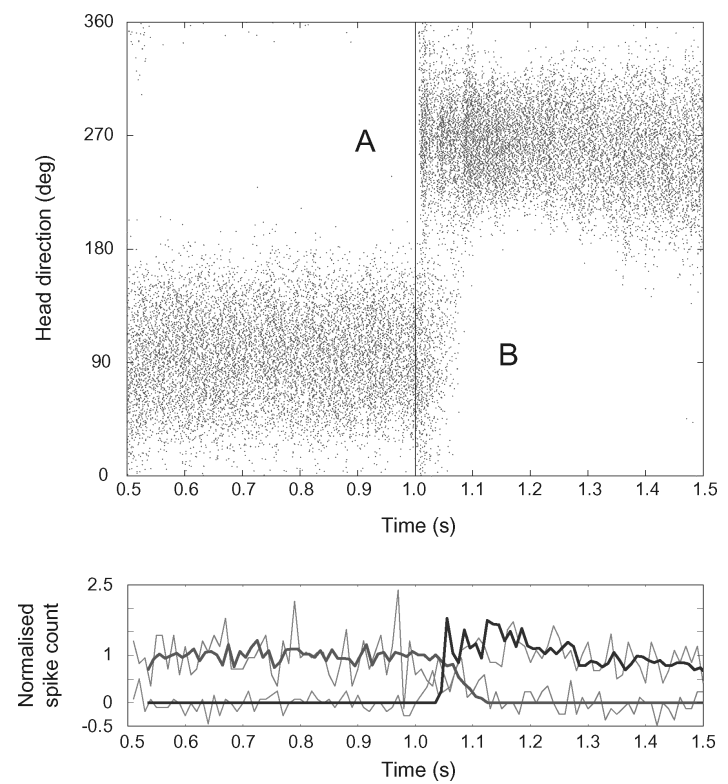

Figure 12. The HD model responds to reorienting external stimuli by rapidly relocating the bump of activity defined by the discharges of all LMN units. Top: Rastergram of the spikes emitted by LMN units over time. Each dot represents one spike. For $t<1 \mathrm{~s}$ the system has a self-sustained tuned state centered at $\bar{\theta} \approx 90^{\circ}$; at $t=1 \mathrm{~s}$ a strong external input is applied to the system at $\theta=180^{\circ}$ and provokes the state transition. The new bump of activity (A) in turn extinguishes the existing bump (B), through an increase in inhibition from DTN. Bottom: Spike peri-event histograms (bins of $10 \mathrm{~ms}$ ) for the sub-population of HD cells that return to baseline activity after the reorientation event (thick red line: simulation; thin orange line: experimental data) and for sub-group of cells that become active after the stimulus onset (thick blue line: simulation; thin blue line: experimental data). The simulation data has been shifted in time by $25 \mathrm{~ms}$ to maximize the overlap between the two sets of curves. Note the good agreement between simulation and theory.

with preferred orientation close to the old bump location, and those with preferred orientation close to the new bump location, for both simulation and experimental data. The normalization was done as follows: we computed the average firing rates in the intervals $[-500,-100] \mathrm{ms}$ and $[100,500] \mathrm{ms}$ where $0 \mathrm{~ms}$ corresponds to the reorienting event. After normalization, these firing rate averages became 0 and 1 . As shown in Fig. 12B, there is a good agreement between simulation and experiment, provided the simulation data is shifted by $25 \mathrm{~ms}$. This temporal shift of $25 \mathrm{~ms}$ thus gives an estimate of the latency for the visual inputs to reach the HD cell system. Finally, note that the relative time course of the two events (appearance of the new bump followed by extinction of the previous bump) is independent of the implementation of the stimulus, provided it is strong enough to provoke the appearance of a new bump. However, the quantitative time course depends on the details of the external inputs.

\section{Discussion}

Self-sustaining persistent activity in the brain has been postulated to mediate working memory functions (see e.g. Wang, 2001). The discharge of head direction (HD) cells constitutes an example of persistent neuronal activity that might serve an ongoing memory trace of the allocentric orientation of the rat, which could be used for navigation purposes (Wiener and Arleo, 2004).

In this paper, we have studied an attractor-integrator network model without recurrent excitation, using the architecture first proposed by Song and Wang (2003). Our paper provides the first analytical investigation of this architecture using simplified networks of threshold-linear neurons. The mathematical analysis provides the boundaries of the parameter space for which direction selectivity is present, and those for which the network is able to integrate accurately angular velocity information. In particular, the saturation velocity was shown to depend critically on the angular offset in the projections between LMN and DTN networks.

The simulations of Section 4 confirm the validity of this scenario in a network of spiking neurons, which was already demonstrated by Song and Wang (2003). Our simulation results are complementary from those of Song and Wang $(2003,2004)$ : they showed how the network dynamics is influenced by the NMDA/AMPA ratio, and how the update of the HD signal by external cues can take the form of either a continuous rotation of the network state or a discontinuous jump to the new HD depending on the distance between the old and new HDs and the strength of the external cue (consistent with previous studies in networks with simpler architectures, e.g. Compte et al., 2000). Here, we have shown how the network dynamics is influenced by the mutual inhibitory connections in DTN, and how the offset in the projections from DTN to LMN control the saturation velocity above which the system is no longer able to integrate accurately. Finally, both our work and the work of Song and Wang (2004) have demonstrated that a system with such an architecture is able to perform rather well when real angular velocity data is applied to it, and that it is able to update following a strong reorienting signal.

The model presented here is consistent with both anatomical and neurophysiological findings currently 
available. From the anatomical point of view, the structures we have considered (i.e., LMN and DTN) are known to be critically involved in the maintenance of the HD signal, and their inter-connectivity as well as the nature of the projections (i.e., excitatory vs inhibitory) have been documented (see e.g., Sharp et al., 2001). From the neurophysiological point of view, the tuning curves we observe are quantitatively similar to the tuning curves recorded in the rat HD cell system. HD cells are numerous in the LMN (Blair et al., 1998; Stackman and Taube, 1998). In the DTN, the picture is less clear, because few electrophysiological recordings are available in this area (Sharp et al., 2001; Bassett and Taube, 2001b). HD cells in the DTN typically have broader tuning curves than in other structures, and some cells are modulated by both angular velocity and head direction (AV-by-HD cells) and are therefore functionally similar to the 'DTN cells' in the model we have studied (Sharp et al., 2001). These cells often have linear dependence of the firing rate vs angular velocity, as in our model. In fact, these 'AV' tuning curves can be extrapolated to lead to zero firing rate at around $1000^{\circ}$ per second, which our model predicts to be the order of magnitude of the saturation velocity. However, most recorded cells in DTN show a clear AV but not HD dependence. This type of cell is not present in our model. Hence, more data is needed in order to conclude whether the mechanisms studied here are necessary and/or sufficient to maintain and integrate the HD signal.

Our analytical results are a generalization of previous results in simpler models for maintenance/ integration in the HD system (Zhang, 1996; Xie et al., 2002). Our model includes a more realistic architecture for the HD system, compared to the two-population models of Zhang (1996) and Xie et al. (2002). In particular, in the two population model of Xie et al. (2002), 'left' and 'right' populations are connected directly together in an excitatory fashion. Both our model and that of Xie et al. (2002) have a saturation velocity that depends only on the angular offset of the connectivity $\alpha$ and the synaptic time constant $\tau$, though the dependence on $\alpha$ is different: $\tan \alpha$ in Xie et al. (2002) vs $(1-\cos (\alpha)) /$ $\sin \alpha$ in our model. The range of saturation velocities is typically of the order of $1000^{\circ}$ in both models.

Are there computational advantages of the architecture we studied here? One possible advantage is that the lack of recurrent excitatory connections permits to stabilize a selective attractor with relatively low firing rates for a wide range of parameters, unlike networks with recurrent excitation, where selective persistent ac- tivity at low rates occurs typically in a narrow parameter range.

In the model studied here, sustained persistent activity depends on the network interactions. A weak point of the model is the perfect homogeneity of the connectivity structure, with translation invariance along the circle of preferred orientations. Inhomogeneities are known to disrupt continuous attractors in a small number of discrete attractors. However, additional single cell properties can reinforce the stability of the attractor network (Camperi and Wang, 1998; Koulakov et al., 2002). In particular, known electrophysiological properties of LMN neurons could stabilize the selective sustained discharge of HD cells (Llinás and Alonso, 1992). More theoretical and experimental studies are necessary to clarify the role of single cell properties in maintenance and/or integration of the HD signal.

\section{Appendix A}

\begin{tabular}{ll}
\hline Abbreviations & \multicolumn{1}{c}{ Full name } \\
\hline ADN & Anterodorsal thalamic nucleus \\
DTN & Dorsal tegmental nucleus \\
HD & Head direction \\
LMN & Lateral mammillary nucleus \\
PoSC & Postsubiculum (dorsal presubiculum)
\end{tabular}

\begin{tabular}{|c|c|}
\hline Variables & Definition \\
\hline \multicolumn{2}{|c|}{ Both types of models } \\
\hline$\theta$ & Animal's head direction (deg) \\
\hline$\theta_{i}$ & Preferred direction (deg) of neuron $i$ \\
\hline$v$ & Bump angular velocity \\
\hline$v_{h}$ & Head angular velocity \\
\hline$I_{E, I}$ & $\begin{array}{l}\text { Background external input to excitatory/ } \\
\text { inhibitory network }\end{array}$ \\
\hline$I_{l, r}$ & $\begin{array}{l}\text { Velocity-dependent external input to left/right } \\
\text { DTN }\end{array}$ \\
\hline$H(\cdot)\left(H_{0}, H_{1}\right)$ & $\begin{array}{l}\text { Footprint of excitatory connections from LMN } \\
\text { to DTN (average, modulation) }\end{array}$ \\
\hline$K(\cdot)\left(K_{0}, K_{1}\right)$ & $\begin{array}{l}\text { Footprint of inhibitory connections from DTN } \\
\text { to LMN (average, modulation) }\end{array}$ \\
\hline$L_{0}$ & Mutual inhibition between DTN networks \\
\hline$\alpha$ & $\begin{array}{l}\text { Angular offset of the projections from DTNs } \\
\text { to LMN (deg) }\end{array}$ \\
\hline
\end{tabular}




\section{(Continued).}

\section{Rate models, Sections 2.3}

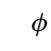

$\begin{array}{ll}f_{A} & \begin{array}{l}\text { Mean firing rate of population } A \\ \tau\end{array} \\ s_{A 0}, s_{A 1} & \begin{array}{l}\text { Synaptic time constant } \\ \text { Average and first Fourier component } \\ \text { of synaptic activation variables } \\ \text { Center of mass of activation variable }\end{array} \\ \psi_{A} & \begin{array}{l}\text { Location of bump in firing rate profile } \\ \phi_{A} \\ \theta_{A}\end{array} \\ \text { Width of the bump of population } A\end{array}$

Spiking neuron model, Section 4

\begin{tabular}{|c|c|}
\hline$N_{E}, N_{I}$ & $\begin{array}{l}\text { Number of excitatory/inhibitory } \\
\text { directional neurons }\end{array}$ \\
\hline$\sigma_{E}, \sigma_{I}$ & Width of footprints (deg) \\
\hline$V(t), I(t)$ & $\begin{array}{l}\text { Neuron's membrane potential }(\mathrm{mV}) \text {, } \\
\text { input current (nA) }\end{array}$ \\
\hline$V_{\text {leak }}, V_{\text {threshold }}, V_{\text {reset }}$ & $\begin{array}{l}\text { Resting, firing threshold, reset } \\
\text { membrane potential }(\mathrm{mV})\end{array}$ \\
\hline$V_{E}$ and $V_{I}$ & $\begin{array}{l}\text { Reversal potential for excitatory and } \\
\text { inhibitory synapses }\end{array}$ \\
\hline$C$ & Neuron's membrane capacitance $(\mathrm{nF})$ \\
\hline$g$ & Membrane conductance (nS) \\
\hline$g_{\mathrm{GABA}}, g_{\mathrm{AMPA}}, g_{\mathrm{NMDA}}$ & $\begin{array}{l}\text { GABA, AMPA, NMDA individual } \\
\text { synaptic conductances }(\mathrm{nS})\end{array}$ \\
\hline$s$ & Synaptic activation variable \\
\hline$t_{\mathrm{ref}}$ & Refractory period (ms) \\
\hline $\bar{\theta}$ & $\begin{array}{l}\text { Estimated head direction, i.e. center } \\
\text { of mass of the activity profile, (deg) }\end{array}$ \\
\hline$\theta_{R}$ & $\begin{array}{l}\text { Allocentric direction of the } \\
\text { reorienting stimulus (deg) }\end{array}$ \\
\hline$\sigma_{R}$ & $\begin{array}{l}\text { Width of the gaussian profile of the } \\
\text { external stimulus } R \text { (deg) }\end{array}$ \\
\hline$\epsilon$ & Mean tracking error function \\
\hline$\beta$ & $\begin{array}{l}\text { Angle of rotation of the reorienting } \\
\text { stimulus (deg) }\end{array}$ \\
\hline$R(t)$ & $\begin{array}{l}\text { Exponential function determining the } \\
\text { intensity of the external stimulus }\end{array}$ \\
\hline
\end{tabular}

\section{References}

Allen GV and Hopkins DA (1988) Mamillary body in the rat: A cytoarchitectonic, Golgi, and ultrastructural study. J. Comp. Neurol. 275(1): 39-64.

Allen GV, Hopkins DA (1989) Mamillary body in the rat: Topography and synaptology of projections from the subicular complex, prefrontal cortex, and midbrain tegmentum. J. Comp. Neurol. 286: 311-336.
Allen GV, Hopkins DA (1990) Topography and synaptology of mammillary body projections to the mesencephalon and pons in the rat. J. Comp. Neurol. 301: 214-231.

Amari SI (1977) Dynamics of pattern formation in lateral-inhibition type neural fields. Bio. Cyber. 27: 77-87.

Arleo A, Gerstner W (2001) Spatial orientation in navigating agents: Modeling head-direction cells. Neurocomput. 38-40(1-4): 10591065.

Bassett JP, Taube JS (2001a) Lesions of the dorsal tegmental nucleus of the rat disrupt head direction cell activity in the anterior thalamus. Soc. Neurosci. Abst. 27: 852.29.

Bassett JP, Taube JS (2001b) Neural correlates for angular head velocity in the rat dorsal tegmental nucleus. J. Neurosci. 21(15): 5740-5751.

Battaglia FP, Treves A (1998) Attractor neural networks storing multiple space representations: A model for hippocampal place fields. Phys. Rev. E 58: 7738-7753.

Ben-Yishai R, Hansel D, Sompolinsky H (1997) Traveling waves and the processing of weakly tuned inputs in a cortical network module. J. Comput. Neurosci. 4: 57-77.

Ben-Yishai R, Lev Bar-Or R, Sompolinsky H (1995) Theory of orientation tuning in visual cortex. Proc. Natl. Aca. Sci. USA 92: 3844-3848.

Blair H, Cho TJ, Sharp PE (1998) Role of the lateral mammillary nucleus in the rat head direction circuit: A combined single unit recording and lesion study. Neuron 21: 1387-1397.

Blair HT, Sharp PE (1995) Anticipatory head direction signals in anterior thalamus: Evidence for a thalamocortical circuit that integrates angular head motion to compute head direction. J. Neurosci. 15(9): 6260-6270.

Brunel N (2003) Dynamics and plasticity of stimulus-selective persistent activity in cortical network models. Cerebral Cortex 13: 1151-1161.

Brunel N, Wang X-J (2001) Effects of neuromodulation in a cortical network model of object working memory dominated by recurrent inhibition. J. Comput. Neurosci. 11: 63-85.

Camperi M, Wang X-J (1998) A model of visuospatial short-term memory in prefrontal cortex: Recurrent network and cellular bistability. J. Comput. Neurosci. 5: 383-405.

Chen LL, Lin L, Green EJ, Barnes CA, McNaughton BL (1994) Head-direction cells in the rat posterior cortex. I. Anatomical distribution and behavioral modulation. Exp. Brain Res. 101: 823.

Cho J, Sharp PE (2001) Head direction, place, and movement correlates for cells in the rat retrosplenial cortex. Behav. Neurosci. 115(1): 3-25.

Compte A, Brunel N, Goldman-Rakic PS, Wang X-J (2000) Synaptic mechanisms and network dynamics underlying spatial working memory in a cortical network model. Cerebral Cortex 10: 910923.

Degris T, Sigaud O, Wiener SI, Arleo A (2004) Rapid response of head direction cells to reorienting visual cues: A computational model. Neurocomput. 58-60C: 675-682.

Ermentrout GB (1998) Neural networks as spatio-temporal patternforming systems. Rep. Prog. Phys. 61: 353-430.

Georgopoulos AP, Schwartz A, Kettner RE (1986) Neuronal population coding of movement direction. Science 233: 14161419.

Gonzalo-Ruiz A, Alonso A, Sanz JM, Llińas RR (1992) Afferent projections to the mammillary complex of the rat, with special 
reference to those from surrounding hypothalamic regions. J. Comp. Neurol. 321: 277-299.

Goodridge JP, Dudchenko PA, Worboys KA, Golob EJ, Taube JS (1998) Cue control and head direction cells. Behav. Neurosci. 112(4): 749-761.

Goodridge JP, Taube JS (1995) Preferential use of landmark navigational system by head direction cells in rats. Behav. Neurosci. 109: 49-61.

Goodridge JP, Taube JS (1997) Interaction between the postsubiculum and anterior thalamus in the generation of head direction cell activity. J. Neurosci. 17(23): 9315-9330.

Goodridge JP, Touretzky DS (2000) Modeling attractor deformation in the rodent head-direction system. J. Neurophysiol. 83(6): 34023410 .

Gutkin BS, Laing CR, Colby CL, Chow CC, Ermentrout GB (2001) Turning on and off with excitation: The role of spike time asynchrony and synchrony in sustained neural activity. J. Comput. Neurosci. 11: 121-134.

Hansel D, Sompolinsky H (1998) Modeling feature selectivity in local cortical circuits. In: C Koch, I Segev, eds. Methods in Neuronal Modeling, 2nd ed. MIT press, Cambridge, MA.

Jahr CE, Stevens CF (1990) Voltage dependence of NMDA-activated macroscopic conductances predicted by single-channel kinetics. J. Neurosci. 10: 3178-3182.

Kali S, Dayan P (2000) The involvement of recurrent connections in area CA3 in establishing the properties of place fields: A model. J. Neurosci. 20(19): 7463-7477.

Knierim JJ, Kudrimoti HS, McNaughton BL (1998) Interactions between idiothetic cues and external landmarks in the control of place cells and head direction cells. J. Neurophysiol. 80: 425-446.

Koulakov AA, Raghavachari S, Kepecs A, Lisman JE (2002) Model for a robust neural integrator. Nature Neurosci. 5: 775782.

Laing CR. Chow CC (2001) Stationary bumps in networks of spiking neurons. Neural Comput. 13: 1473-1494.

Liu R, Chang L, Wickern G (1984) The dorsal tegmental nucleus: An axoplasmic transport study. Brain Res. 310: 123132.

Llinás RR, Alonso A (1992) Electrophysiology of the mammillary complex in vitro. I. Tuberomammillary and lateral mammillary neurons. J. Neurophysiol. 68(4): 1307-1320.

Lukashin AV, Georgopoulos AP (1993) A dynamical neural network model for motor cortical activity during movement: Population coding of movement trajectories. Biol. Cyber. 69: 517-524.

Matthews BL, Campbell KA, Deadwyler SA (1988) Rotational stimulation disrupts spatial learning in fornix-lesioned rats. Behav. Neurosci. 102: 35-42.

McNaughton BL, Chen LL, Markus EJ (1991) Dead reckoning, landmark learning, and the sense of direction: A neurophysiological and computational hypothesis. J. Cog. Neurosci. 3: 190-202.

Mizumori SJY, Williams JD (1993) Directionally selective mnemonic properties of neurons in the lateral dorsal nucleus of the thalamus of rats. J. Neurosci. 13: 40154028.

Ranck JBJ (1984) Head-direction cells in the deep cell layers of dorsal presubiculum in freely moving rats. Soc. Neurosci. Abst. 10: 599 .

Redish AD, Elga AN, Touretzky DS (1996) A coupled attractor model of the rodent head direction system. Network 7(4): 671685.
Redish AD, Touretzky DS (1997) Cognitive maps beyond the hippocampus. Hippocampus 7(1): 15-35.

Rubin J, Terman D, Chow C (2001) Localized bumps of activity sustained by inhibition in a two-layer thalamic network. J. Comput. Neurosci. 10: 313-331.

Samsonovich A, McNaughton BL (1997) Path integration and cognitive mapping in a continuous attractor neural network model. J. Neurosci. 17(15): 5900-5920.

Sharp PE, Blair HT, Cho J (2001) The anatomical and computational basis of the rat head-direction cell signal. Trends Neurosci. 24(5): 289-294.

Sharp PE, Tinkelman A, Cho J (2001) Angular velocity and head direction signals recorded from the dorsal tegmental nucleus of gudden in the rat: Implications for path integration in the head direction cell circuit. Behav. Neurosci. 115(3): 571588.

Shibata H (1987) Ascending projections to the mammillary nuclei in the rat: A study using retrograde and anterograde transport of wheat germ agglutinin conjugated to horseradish peroxidase. J. Comp. Neur. 264: 205-215.

Skaggs WE, Knierim JJ, Kudrimoti HS, McNaughton BL (1995) A model of the neural basis of the rat's sense of direction. In: $G$ Tesauro, DS Touretzky, TK Leen, eds. Advances in Neural Information Processing Systems 7, MIT Press, Cambridge, MA, pp. 173-180.

Somers DC, Nelson SB, Sur M (1995) An emergent model of orientation selectivity in cat visual cortical simple cells. J. Neurosci. 15: 5448-5465.

Song P, Wang X-J (2003) A three-population attractor network model of rodent head direction system. Soc. Neurosci. Abst., 939.3.

Song P, Wang X-J (2004) Time integration by moving 'hill of activity': A spiking neural model without recurrent excitation of the head-direction system. To appear in J. Neurosci.

Stackman RW, Taube JS (1998) Firing properties of rat lateral mammillary single units: Head direction, head pitch, and angular head velocity. J. Neurosci. 18(21): 90209037.

Taube JS (1995) Head direction cells recorded in the anterior thalamic nuclei of freely moving rats. J. Neurosci. 15(1): 70-86.

Taube JS (1998) Head direction cells and the neurophysiological basis for a sense of direction. Prog. Neurobiol. 55: 225256.

Taube JS, Bassett JP (2003) Persistent neural activity in head direction cells. Cerebral Cortex 13: 1162-1172.

Taube JS, Muller RU (1998) Comparisons of head direction cell activity in the postsubiculum and anterior thalamus of freely moving rats. Hippocampus 8: 87-108.

Taube JS, Muller RI, Ranck JBJ (1990a) Head direction cells recorded from the postsubiculum in freely moving rats. I. Description and quantitative analysis. J. Neurosci. 10: 420435.

Taube JS, Muller RI, Ranck JBJ (1990b) Head direction cells recorded from the postsubiculum in freely moving rats. II. Effects of environmental manipulations. J. Neurosci. 10: 436447.

Tsodyks M, Sejnowski T (1995) Associative memory and hippocampal place cells. Int. J. Neur. Syst. 6: 81-86.

Wang X-J (2001) Synaptic reverberation underlying mnemonic persistent activity. Trends Neurosci. 24(8): 455-463. 
Wiener SI (1993) Spatial and behavioral correlates of striatal neurons in rats performing a self-initiated navigation task. J. Neurosci. 13: 3802-3817.

Wiener SI, Arleo A (2004) Persistent activity in limbic system neurons: Neurophysiological and modeling perspectives. J. Physiol. Paris 97(4-6): 547-555.

Wirtshafter D, Stratford TR (1993) Evidence for GABAergic projections from the tegmental nuclei of Gudden to the mammillary body in the rat. Brain Res. 630: 188-194.

Xie X, Hahnloser RHR, Seung HS (2002) Double-ring network model of the head direction system. Phys. Rev. E 66:
041902-1-9.

Zhang K (1996) Representation of spatial orientation by the intrinsic dynamics of the head-direction cell ensemble: A theory. J. Neurosci. 16(6): 2112-2126.

Zugaro MB, Arleo A, Berthoz A, Wiener SI (2003) Rapid spatial reorientation and head direction cells. J. Neurosci. 23(8): 3478 3482 .

Zugaro MB, Berthoz A, Wiener SI (2001) Background, but not foreground, spatial cues are taken as references for head direction responses by rat anterodorsal thalamus neurons. J. Neurosci. 21: $\mathrm{RC} 154(1-5)$. 